\title{
Comment réduire la fracture spatiale ? Une application en Ile-de-France
}

NATHALIE GEORGES, YANNICK L’HORTY, FLORENT SARI

TEPP - Travail, Emploi et Politiques Publiques - FR CNRS 3126 


\title{
Comment réduire la fracture spatiale ? Une application en Île-de-France
}

\author{
Nathalie GEORGES ${ }^{1}$, Yannick L'HORTY² ${ }^{2}$ Florent SARI ${ }^{3}$
}

\section{RÉSUMÉ}

Nous estimons sur des données communales franciliennes un modèle à erreurs spatialement corrélées afin d'expliquer les disparités spatiales de durées de chômage. Nous mobilisons des indicateurs représentatifs du dynamisme de l'emploi local, du mésappariement entre la localisation des emplois et des demandeurs d'emplois, du mésappariement entre les compétences demandées par les entreprises et celles offertes par les demandeurs d'emplois, de la ségrégation résidentielle et du logement. Nous utilisons des méthodes qui permettent d'évaluer la portée de chacune de ces explications et de déterminer lesquelles sont les plus importantes à considérer du point de vue de la décision publique. Les résultats montrent que les efforts devraient être accentués sur les problèmes de mésappariement en termes de compétences et de localisation géographique des demandeurs d'emplois.

Mots-clefs : Modèle d'appariement, fracture spatiale, chômage, politiques publiques.

Classification JEL : C41, J64, R1.

\footnotetext{
${ }^{1}$ ENSAE-PARISTECH et TEPP (FR n³435), , 5 boulevard Descartes, Champs sur Marne 77454 Marne la Vallée cedex 2, nathalie.georges@ensae-paristech.fr .

${ }^{2}$ Université Paris-Est, ERUDITE et TEPP (FR CNRS n³435), 5 boulevard Descartes, Champs sur Marne 77454 Marne la Vallée cedex 2, Yannick.lhorty@univ-mlv.fr.

3 Université Paris-Est, ERUDITE et TEPP (FR CNRS n³435), 5 boulevard Descartes, Champs sur Marne 77454 Marne la Vallée cedex 2, florent.sari@univ-mlv.fr
} 


\section{Introduction}

Menées principalement sur données américaines, de nombreuses études empiriques confirment le rôle joué par la distance physique entre la localisation des emplois et celle des travailleurs dans la difficulté à sortir du chômage, suivant l'hypothèse proposée initialement par Kain (1968). Résider à proximité d'un bassin d'emploi réduit la durée du chômage en diminuant les coûts de transports et les coûts de prospection lors d'une recherche d'emploi (Rogers, 1997 ; Immergluk, 1998 ; Brueckner et Martin, 1997). Pour autant, d'autres facteurs sont mis en avant. Les problèmes de mésappariement locaux entre les compétences offertes et demandées jouent un rôle prépondérant selon Hellerstein et alii (2008), tandis que les effets de pairs et de réseaux sont privilégiés par les travaux plus anciens de Crane (1991) ou O’Reagan (1993).

Les études menées sur données françaises suggèrent également que l'éloignement physique entre la localisation des emplois et celle des travailleurs explique en partie la géographie du chômage. Depuis les premiers résultats obtenus sur données françaises par Cavaco et Lesueur (2002), la distance à l'emploi a permis de rendre compte de la courbe en U du chômage observée autour de Paris (Duguet et alii, 2009), même si des phénomènes de ségrégation spatiale ont aussi un rôle à jouer dans l'analyse des différences locales dans l'accès à l'emploi (Gobillon et Selod, 2007). Une fois contrôlée de la structure de la main-d'œuvre au niveau régional, on observe des différences marquées de durées de chômage pour des communes contigües, ainsi que des «grappes de territoires » homogènes qui ne s'expliquent pas par les caractéristiques sociodémographiques des chômeurs (Duguet et alii, 2009). Il faut alors trouver des explications alternatives à une simple approche par la composition de l'offre de travail pour justifier ce type de structure spatiale.

Du point de vue des politiques publiques, trois voies sont ouvertes pour tenter de réduire les disparités locales d'exposition au chômage de longue durée (Ihlanfeldt et Sjoquist, 1998) : rapprocher les emplois des travailleurs, les travailleurs des emplois ou assurer une meilleure connexion entre les deux. Une subvention publique des transports (Kawabata, 2003 ; Holzer et alii, 2003), ou une aide ciblée à la motorisation des ménages (Raphael et Stoll, 2001), peuvent limiter le mésappariement en termes de localisation et accélérer la sortie du chômage. Néanmoins, les leviers qui permettent de réduire la fracture spatiale et ceux qui contribuent à réduire le chômage constituent deux ensembles disjoints dans un modèle d'économie urbaine avec mésappariement des qualifications offertes et demandées, selon l'étude de Gavrel et alii (2012).

Notre objectif est de rendre compte des disparités spatiales de durée du chômage en intégrant la demande locale de travail à l'aide d'un modèle de flux, où celles-ci sont notamment expliquées par le rythme des créations et destructions d'emplois, estimé sur la base d'indicateurs agrégés au niveau de la commune. L'originalité de ce travail est double. D'une part, nous proposons d'aborder cette question avec des indicateurs de flux (estimés sur des sources administratives exhaustives, alors que la plupart des études qui analysent les déterminants spatiaux du chômage ${ }^{4}$ mobilisent essentiellement des données d'enquêtes et des indicateurs de stocks (taux de chômage, par exemple). D'autre part, nous introduisons dans la modélisation des indicateurs locaux issus de sources d'entreprises qui mesurent la démographie des emplois, tout en testant les effets d'un mauvais appariement lié à la distance entre domicile et emploi, les effets d'un mauvais appariement lié aux qualifications des travailleurs, et les effets d'interactions sociales liés au voisinage. Globalement, nos résultats

\footnotetext{
${ }^{4}$ Voir notamment : Gaschet et Gaussier, 2003 ; Dujardin et alii. (2004), Gobillon et Selod (2007) etc.
} 
indiquent que les trois effets jouent conjointement et qu'il est utile de tenir compte de la demande de travail pour expliquer les disparités géographiques de retour à l'emploi.

Après avoir présenté les données mobilisées pour notre analyse, nous présentons la construction des variables et quelques statistiques descriptives associées. Nous exposons ensuite la méthode économétrique retenue, ainsi que les résultats des différentes estimations. Finalement, la dernière section conclut.

\section{Les données}

Dans cette section, nous présentons les différentes bases de données retenues dans notre analyse. Celles-ci nous permettent de créer la principale variable à expliquer de notre modèle (la durée de chômage communale) ainsi que les différentes variables explicatives. Nous rappelons que notre objectif est de rendre compte des disparités spatiales de durée du chômage à partir d'indicateurs locaux qui mesurent la démographie des emplois, le mauvais appariement lié à la distance entre domicile et emploi, le mauvais appariement lié aux qualifications des travailleurs et les effets d'interactions sociales liés au voisinage.

\section{Fichier Historique Statistique de Pôle Emploi}

Les durées individuelles de chômage du point de vue des demandeurs d'emploi sont délimitées par les sorties du chômage dont la mesure pose un problème de définition et d'observation. Pour les dénombrer, nous utilisons donc la version exhaustive du Fichier Historique Statistique (FHS) de Pôle Emploi qui est une source administrative permettant de suivre les parcours individuels des demandeurs d'emploi en enregistrant tous les événements successifs depuis leur première inscription à Pôle Emploi. Cependant, ce fichier de gestion renseigne mal les motifs de sorties et ne couvre pas les parcours des demandeurs une fois qu'ils ont repris un emploi ou lorsqu'ils ne sont plus inscrits à Pôle Emploi. Or, si le retour à un emploi implique très généralement une sortie des fichiers de Pôle Emploi, toutes les sorties du fichier ne sont pas des retours à l'emploi.

Afin de pouvoir suivre les demandeurs d'emploi dans le temps, sur une durée suffisamment longue, nous nous limitons à une cohorte de personnes qui se sont inscrites entre le ler juillet 2001 et le 30 juin 2002. La date du 1er juillet 2001 a été retenue car elle correspond à l'entrée en vigueur d'un nouveau système d'indemnisation du chômage. On étudie ainsi une période homogène du point de vue de l'indemnisation. Le fichier que nous utilisons est la version du FHS mis à jour jusqu'au 31 mars 2006. Nous suivons la cohorte de chômeurs sur près de cinq ans. Nous calculons ainsi des durées de chômage au niveau de la commune pour tout code postal où résident au moins 100 chômeurs (cf. Duguet et alii, [2007] et [2009]).

\section{Les Déclarations Annuelles de Données Sociales}

Pour rendre compte de la démographie locale de l'emploi, des indicateurs de flux d'emplois ont été construits à partir des Déclarations Annuelles de Données Sociales (DADS), formalité déclarative à laquelle doit s'astreindre toute entreprise employant des salariés. Les DADS forment donc une source exhaustive d'informations sur les entreprises et leurs salariés, à l'exception des secteurs agricole, de la fonction publique d'Etat et des services domestiques. Les DADS contiennent des informations sur les établissements (SIRET, secteur d'activité, effectif au 31 décembre en équivalent temps plein); et sur les salariés (sexe, âge, poste, temps de travail, statut, PCS, rémunération). La base utilisée pour notre étude contient 346545 
établissements présents en Île-de-France entre 2002 et 2005, dont 86342 renseignés aux quatre dates de l'enquête. Elles sont rattachées à l'une des 1149 communes d'île-de-France.

\section{Le recensement de la population de l'INSEE (1999)}

Nous mobilisons également des données du recensement de la population produit par l'Insee et datant de 1999. Le recensement contient des informations à l'échelle des 1300 communes qui composent la région Ile-de-France. Il offre des informations sur les logements, les individus et les ménages résidents. Il permet de savoir les types de logements présents dans le quartier, la taille des immeubles et leur époque d'achèvement. Il apporte des informations sur les caractéristiques de l'habitat collectif, les résidences principales, les résidences secondaires etc. Concernant les individus, les données du recensement apportent un certain nombre d'informations socio-économiques ou démographiques. Elles permettent de connaître la composition d'une commune par âge, sexe, nationalité et niveau de diplôme, mais aussi de recenser la population totale, la population active, la population inactive ou le nombre de chômeurs. Elles renseignent enfin sur la population active par catégorie socio-professionnelle et par secteur d'activité. Enfin, les informations au niveau du ménage sont relatives à sa composition ou encore au statut d'occupation du logement.

\section{La variable à expliquer : la durée du chômage}

\section{Indicateurs et conventions administratives}

Pour mesurer la durée locale du chômage, nous nous intéressons à l'ensemble des chômeurs inscrits à Pôle Emploi, qu'ils soient ou non indemnisés. Il s'agit là d'une définition large du chômage qui comprend l'ensemble des demandes d'emploi en fin de mois des catégories 1, 2, 3 et $6,7,8$. Cette définition prend en compte les chômeurs qui n'exercent pas d'activité réduite (catégories 1 à 3 ) et les demandeurs d'emploi qui exercent une activité réduite de plus de 78 heures par mois, qu'ils recherchent un CDI à temps complet (catégorie 6), à temps partiel (catégorie 7), ou un autre contrat (catégorie 8).

Nous considérons qu'un individu sort du chômage dès lors qu'il est sorti des listes de Pôle Emploi. La convention administrative des «sorties de listes » est extensive : elle englobe tous les motifs de sortie quel que soit le motif déclaré, y compris les absences au contrôle et les radiations administratives. Bien que cette convention exagère potentiellement l'ampleur du retour à l'emploi, nous la retenons car elle constitue une référence usuelle, souvent utilisée dans les publications officielles qui suivent la conjoncture du marché du travail ${ }^{5}$.

\section{Estimations toutes choses égales par ailleurs à l'aide de modèles de durée}

Pour modéliser la durée du chômage, nous avons retenu une spécification à la Weibull. Selon ce modèle, le taux de sortie du chômage est une fonction du temps passé au chômage et dépend aussi des caractéristiques de l'individu, telles que l'âge, le sexe, ou le niveau de diplôme. L'avantage est de prendre en compte explicitement les effets des caractéristiques individuelles par l'introduction de variables explicatives dans la régression. L'inconvénient est de supposer que les chances de sortie sont strictement proportionnelles à l'ancienneté de chômage.

\footnotetext{
${ }^{5}$ Les sorties de listes font l'objet d'un suivi régulier de la part du Ministère du Travail, la DARES y consacrant chaque trimestre un numéro de sa collection "Premières Informations et Premières Synthèses".
} 
Nous avons cependant vérifié que le recours à d'autres techniques d'estimation modifie peu les taux de sortie. Les coefficients estimés avec un modèle de Weibull sont très proches de ceux estimés avec un modèle à hasard constant par intervalles ou avec un modèle de Cox. Dans nos estimations, le paramètre $\alpha$ du modèle de Weibull, qui conditionne la relation entre la durée passée au chômage et les chances d'en sortir à chaque période, est proche de l'unité. Ce cas particulier $(\alpha=1)$ correspond au modèle exponentiel, où la durée de chômage passée n'a pas d'effet sur le taux de sortie instantané du chômage. Dans ce cas, toutes les généralisations habituelles donnent des résultats équivalents.

Pour calculer des taux de sortie et des espérances de durée au niveau communal nous avons fixé un nombre minimal de 100 chômeurs inscrits dans la commune. S'il y a 100 demandeurs d'emploi ou plus dans la commune, nous évaluons nos indicateurs au niveau de cette localité. Dans le cas contraire, nous regroupons la commune avec les autres communes de son code postal dans lesquelles il y a moins de 100 demandeurs d'emploi. Nous évaluons alors nos indicateurs au niveau de ce code postal. S'il y a moins de 100 chômeurs dans le code postal, nous ne calculons pas d'indicateur de sortie du chômage. Les indicateurs locaux sont donc estimés au niveau communal ou à celui du code postal.

L'estimation des modèles nous permet de calculer deux types d'indicateurs au niveau communal. Le premier indicateur, appelé «durée brute», équivaut à une statistique descriptive de retour à l'emploi au niveau de la localité. Elle correspond à la durée moyenne de chômage dans la commune pour les demandeurs d'emploi qui sont entrés au chômage entre juillet 2001 et juin 2002. Pour tenter de séparer les effets de territoire des effets individuels, nous introduisons un deuxième indicateur, appelé «durée nette ». Il s'agit de la durée du chômage que l'on devrait constater si les demandeurs d'emploi avaient les mêmes caractéristiques sur tous les territoires, c'est-à-dire en raisonnant «toutes choses égales par ailleurs ». De façon intuitive, les «durées brutes » sont issues de spécifications où les seules variables explicatives retenues sont des indicatrices communales alors que les «durées nettes » introduisent d'autres variables individuelles de contrôle afin de neutraliser les effets de la composition de la population des demandeurs d'emplois.

Techniquement, les «durées nettes » sont obtenues en estimant un modèle à effet fixe au niveau communal. Ce modèle inclut, en plus des effets fixes locaux, toutes les caractéristiques socio-économiques des demandeurs d'emploi, de sorte qu'il permet d'estimer un effet de territoire «toutes choses égales par ailleurs ». Comme les effets fixes locaux ne sont pas directement interprétables, on présente les résultats sur les durées nettes de la manière suivante : on fixe les variables socio-économiques à une valeur de référence, qui est la moyenne régionale, puis on calcule la durée du chômage qui correspond à cette moyenne en laissant les effets fixes locaux inchangés. Par construction, cette durée nette neutralise toutes les différences de composition socio-économique de la main-d'oeuvre puisque l'on impose la même valeur des variables socioéconomiques à toutes les unités locales. Cette convention permet de faire ressortir l'effet de territoire et de rendre les durées nettes des différentes unités locales directement comparables.

\section{Faits stylisés}

Nous nous intéresserons donc aux «durées nettes » en convention « sorties de liste », dont les résultats sont synthétisés par la carte 1 . Nous constatons que, même corrigées de la composition de la population active, les disparités locales des taux de retour à l'emploi sont fortes en Île-de-France, avec $10 \%$ des communes où la durée de chômage est inférieure à 9,8 mois, et $10 \%$ où elle est supérieure à 13,7 mois. 


\section{Carte 1. Durées nettes de chômage en Île-de-France}

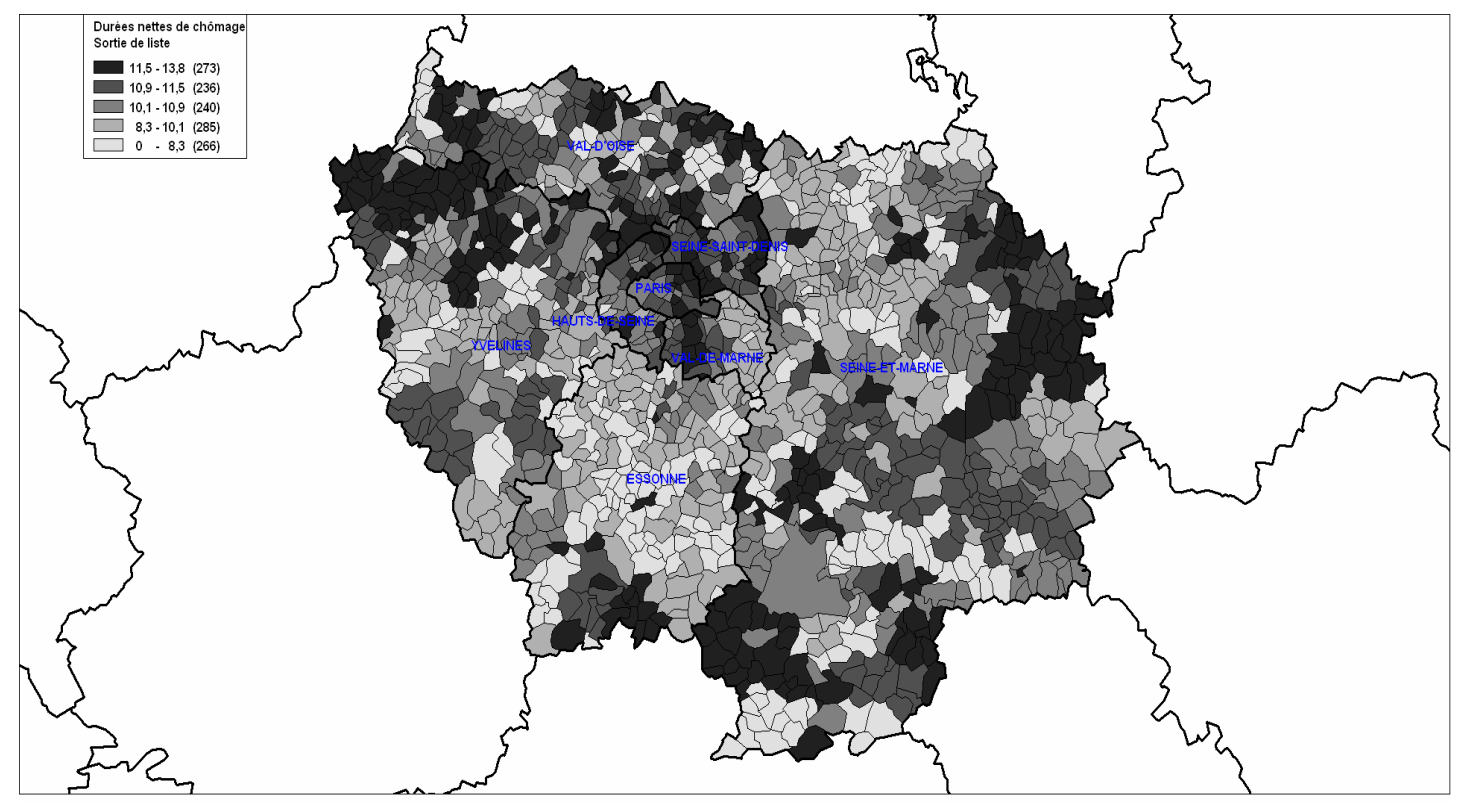

Lecture : Les communes en noir sont celles où les durées de chômage sont les plus élevées. Les communes en gris clair sont celles où les durées sont les plus faibles.

Source : FHS de Pôle Emploi 2006.

Les durées de chômage ne semblent pas distribuées au hasard dans l'espace francilien. D'une part, on relève aussi un phénomène de polarisation: il existe des grappes de communes voisines pour lesquelles les durées moyennes sont proches, parfois uniformément faibles, parfois uniformément élevées. Ce phénomène traduit la présence d'auto-corrélation spatiale et il importera d'en tenir compte dans les estimations. D'autre part, on constate visuellement l'existence d'une structure spatiale particulière, révélant une véritable géographie du chômage. Les durées locales de chômage s'organisent selon un schéma concentrique, avec des durées plus élevées au centre (Paris et première couronne) et à la grande périphérie, et des durées plus faible dans la périphérie intermédiaire.

Cette structure spatiale n'est pas la conséquence d'effets de composition puisque l'on contrôle ici par les caractéristiques socio-démographiques des demandeurs d'emploi. Elle peut traduire des problèmes d'accès aux emplois, des difficultés d'appariements entre les qualifications offertes et demandées, des phénomènes de ségrégation spatiale ou encore les effets de certaines politiques publiques. Pour être en mesure de discuter ces différentes hypothèses, il est nécessaire d'estimer un modèle avec ces différents déterminants qui tienne compte du dynamisme local de l'emploi lié à la démographie des entreprises.

\section{Les variables explicatives}

Nous présentons successivement les différentes variables explicatives (et leur construction) de notre modèle économétrique. Elles renvoient à différents mécanismes identifiés dans la littérature en économie urbaine qui permettent d'expliquer le chômage local. Nous présentons également, pour chacune, quelques statistiques pour la région Île-de-France. 


\section{La mesure du mauvais appariement spatial}

L'hypothèse de mauvais appariement spatial correspond à une déconnection géographique entre la localisation des emplois et celle des chômeurs. Un premier indice peut en être donné avec la proportion de travailleurs qui doit se déplacer pour se rendre sur son lieu de travail. Plus celle-ci est élevée, plus le risque de mauvais appariement spatial est fort, puisque les chômeurs résidant dans ces communes ont alors moins de chances de trouver un emploi à proximité, leur probabilité d'être embauchés augmentant avec leur mobilité. La cartographie de la proportion des actifs qui travaillent dans la zone d'emploi ${ }^{6}$ à laquelle appartient leur commune suggère que les espaces où le retour à l'emploi est le plus rapide sont justement ceux où l'emploi local est le plus développé (le centre des Yvelines, l'est de l'Essonne), alors que d'autres territoires qui souffrent d'un retour à l'emploi difficile ont un faible taux de travailleurs dont l'activité se trouve à proximité (la Seine-Saint-Denis, le nord des Yvelines, l'est de la Seine-et-Marne) ( $c f$. carte 2). Ce constat est un indice en faveur de l'hypothèse de mauvais appariement spatial, puisqu'il suggère que les zones où peu de personnes travaillent sur place sont également celles où il est difficile de sortir rapidement du chômage.

On approxime la distance physique aux emplois à l'aide de deux autres indicateurs résumant les éventuelles difficultés d'accessibilité auxquelles peuvent être confrontés les travailleurs, liées à l'éloignement entre le domicile et le travail. Il s'agit d'une part de la distance moyenne à vol d'oiseau entre le domicile et le travail, et d'autre part de la densité d'emplois accessibles dans un rayon de $20 \mathrm{~km}$ à partir du centroïde des communes ${ }^{7}$ :

$$
\text { Densité d'emplois }_{i}=\frac{\sum_{j} \text { emplois }_{j}}{\sum_{j} \text { population active }_{j}}
$$

où $j$ est l'ensemble des communes dans un rayon de $20 \mathrm{~km}$ autour de la commune $i$.

\footnotetext{
${ }^{6}$ Au sens de la définition de l'Insee.

${ }^{7}$ Cet indice tend à surestimer l'isolement en termes d'accessibilité aux emplois des communes éloignées de Paris. Les travailleurs qui résident dans ces communes périphériques peuvent également travailler dans des régions limitrophes non prises en compte ici, puisque nous ne disposons de données que pour l'île-de-France. Cette limite est inhérente à notre étude, car nous supposons que tous les Franciliens travaillent en Île-de-France alors que des résidents des communes limitrophes peuvent également travailler dans une autre région.
} 


\section{Carte 2. Part des actifs travaillant dans la zone d'emploi de la commune}

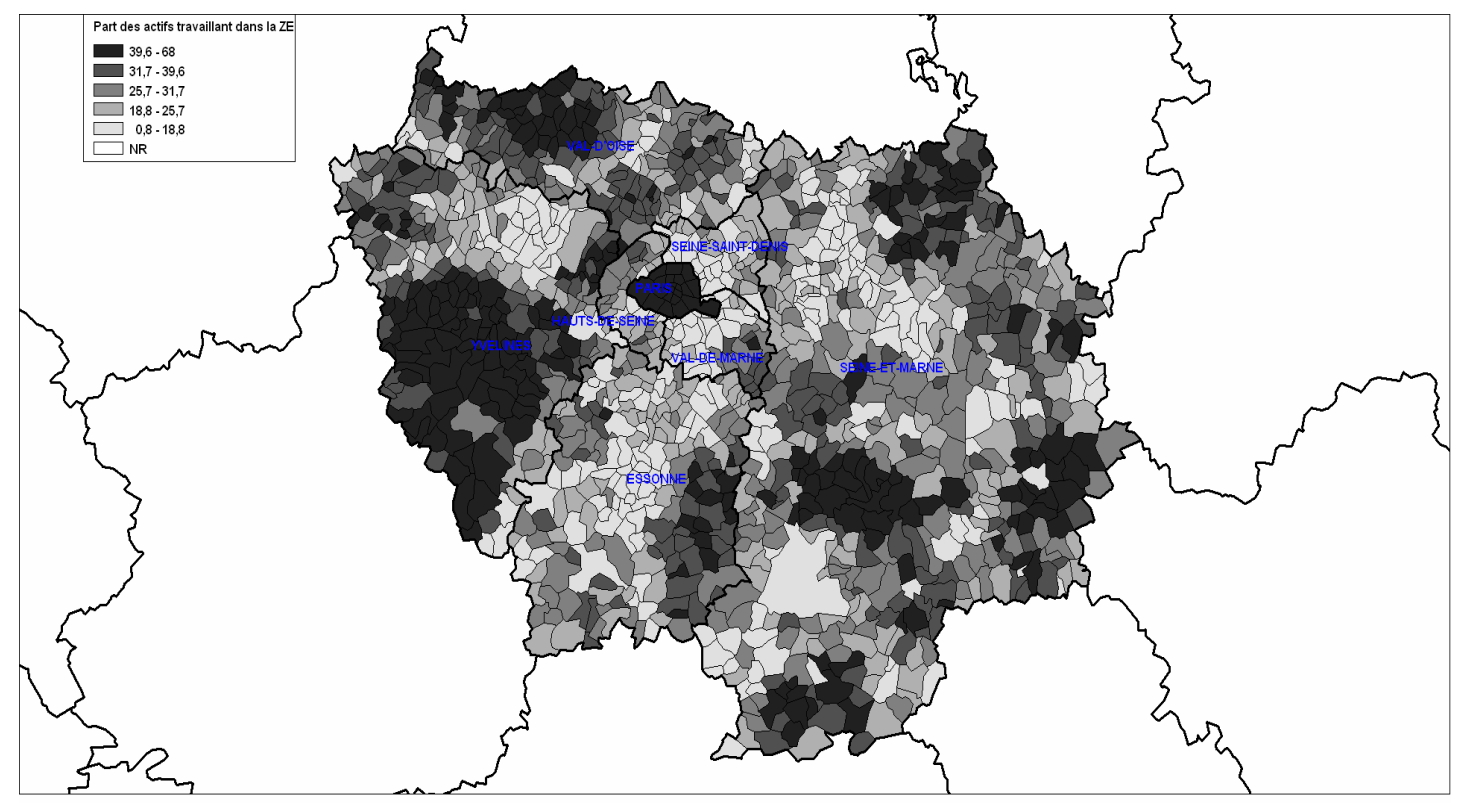

Lecture : Les communes en noir sont celles où la part des habitants qui travaillent dans la même zone d'emploi que la commune de résidence est la plus élevée. Les communes en gris clair sont celles où la part est la plus faible.

Source : Recensement 1999 de l'Insee.

\section{Le dynamisme local de l'emploi}

Outre la localisation des emplois existants, celle des emplois créés ou supprimés est également importante pour explorer l'hypothèse de mauvais appariement spatial. En effet, les zones dynamiques en termes de créations ou de destructions d'emplois sont celles où les chômeurs ont le plus de chances de connaître un chômage de courte durée, car les mouvements de main-d'œuvre permettent un fort turn-over qui profite à ceux qui recherchent un emploi (même si la pérennité de l'emploi trouvé risque alors d'être plus faible). Il est donc intéressant de comparer la démographie des emplois avec la géographie du chômage. Si l'hypothèse de mauvais appariement spatial est vérifiée, on doit constater empiriquement que les entreprises dynamiques se trouvent dans les zones où la sortie du chômage est plus rapide que la moyenne, les travailleurs résidant sur place bénéficiant les premiers de ce dynamisme.

La façon la plus intuitive de décrire le dynamisme des entreprises en termes d'emploi est de mesurer les variations de main-d'œuvre qu'elles subissent d'une période à une autre. En effet, le nombre d'emplois qu'elles créent ou détruisent est un proxy de leur demande de travail sur un intervalle de temps donné. L'approche par les «flux bruts » d'emplois consiste ainsi à comptabiliser les variations des effectifs employés par les entreprises entre deux dates pour lesquelles on connaît le stock total d'employés. Cette approche se heurte cependant à un double écueil : d'une part, elle ne prend en compte que les variations d'emplois liées aux créations et destructions d'emplois au sein des entreprises pérennes, et non le flux des destructions et créations des entreprises elles-mêmes ${ }^{8}$; et d'autre part, elle est limitée par la fenêtre d'observation, ici annuelle, et ne peut rien dire des mouvements de main-d'œuvre infra-

\footnotetext{
${ }^{8}$ Estimées à $35 \%$ respectivement des créations et des destructions d'emploi en France par Duhautois (2002), soit plus de la moitié des mouvements totaux annuels de main-d'œuvre. Nos propres estimations à l'aide d'un panel non cylindré sont mêmes supérieures ( $c f$. infra), alors que Picart (2008) révise fortement à la baisse les flux d'emplois en tenant compte des «fausses mobilités » liées aux modifications de la structure des entreprises.
} 
annuels, pourtant importants dans certains secteurs. En outre on ne mesure pas ici non plus les potentielles modifications de structure au sein des entreprises (mutations internes ou restructurations).

Ces limites posées, la construction d'indicateurs de flux annuels d'emploi inspirés de Davis et Haltiwanger (1990) présente l'avantage de synthétiser l'information contenue dans les données traduisant le processus d'ajustement de l'emploi en indicateurs simples et faciles à interpréter. Les créations brutes d'emplois correspondent aux variations positives entre les effectifs $N$ à deux dates successives, et les destructions brutes d'emplois aux variations négatives. Formellement, le volume des créations brutes d'emplois $C_{c t}$ dans la commune $c$ entre les dates $t-1$ et $t$ est :

$$
C_{c t}=\sum_{e \in C^{+}} \Delta N_{e c t}
$$

où $C^{+}$est le sous-ensemble des entreprises $e$ des communes $c$ pour lesquelles le nombre d'emplois en fin de période est supérieur au nombre d'emplois en début de période d'observation, et $\Delta$ l'opérateur différence entre $t-1$ et $t$. De même, le volume des destructions brutes d'emplois $D_{c t}$ est :

$$
D_{c t}=\sum_{e \in C^{-}}\left|\Delta N_{e c t}\right|
$$

où $C^{-}$est le sous-ensemble des entreprises $e$ des communes $c$ qui ont connu une variation négative de l'emploi au cours de l'année.

On définit la réallocation brute d'emplois $R_{c t}$ comme le volume total des mouvements de main-d'œuvre par commune, soit :

$$
R_{c t}=\sum_{e \in C}\left|\Delta N_{e c t}\right|=C_{c t}+D_{c t}
$$

Pour exprimer ces mêmes chiffres en taux de création (respectivement de destruction ou de réallocation) d'emplois, on les normalise par l'effectif moyen des entreprises de la commune entre les deux dates considérées, soit :

$$
c_{c t}=\frac{C_{c t}}{Z_{c t}}, d_{c t}=\frac{D_{c t}}{Z_{c t}} \text {, et } r_{c t}=\frac{R_{c t}}{Z_{c t}} \text { où } Z_{c t}=\frac{1}{2}\left(N_{c t}+N_{c t-1}\right)
$$

Le taux de croissance nette de l'emploi pour chaque commune correspond alors à la différence entre les taux de création et de destruction :

$$
g_{c t}=c_{c t}-d_{c t}
$$

En moyenne, sur les années 2002-2005, les destructions d'emplois dans les entreprises d' ̂̂lede-France présentes aux trois dates ont été de 6,3\%, légèrement supérieures aux créations (5,6\%, cf. tableau 1). La transition 2002-2003 s'oppose aux suivantes, avec un taux de croissance nette positif, et le plus fort taux de création. 2004-2005 est une année dynamique en termes d'emploi, avec le plus fort taux de réallocation, mais témoigne d'une conjoncture défavorable avec un taux de croissance négatif de 1,8\% et le plus fort taux de destruction d'emplois. 
Tableau 1. Flux bruts d'emplois (2002-2005)

\begin{tabular}{lccccc}
\hline & $\begin{array}{c}\text { Taux de } \\
\text { création }\end{array}$ & $\begin{array}{c}\text { Taux de } \\
\text { destruction }\end{array}$ & $\begin{array}{c}\text { (1) Taux de } \\
\text { réallocation }\end{array}$ & $\begin{array}{c}\text { (2) Taux de } \\
\text { croissance } \\
\text { nette }\end{array}$ & $\begin{array}{c}\text { Corrélation } \\
\text { entre (1) et (2) }\end{array}$ \\
\hline $2002-2003$ & 6,2 & 5,5 & 11,7 & 0,7 & $-0,18^{* * *}$ \\
$2003-2004$ & 4,9 & 6,0 & 10,9 & $-1,1$ & $-0,33^{* * * *}$ \\
$2004-2005$ & 5,7 & 7,5 & 13,2 & $-1,8$ & $-0,39^{* * *}$ \\
\hline Moyenne & 5,6 & 6,3 & 11,9 & $-0,7$ & $-0,28 * * *$ \\
\hline
\end{tabular}

Champ : établissements pérennes de la région Île-de-France.

Lecture : taux en pourcentage, $* * *$ : significatif à 0,01 .

Source : DADS 2002-2005.

Le signe de la corrélation temporelle entre les taux de réallocation et de croissance nette de l'emploi est un indicateur qui nous renseigne sur le principal vecteur d'ajustement de l'emploi. En effet, si la corrélation entre les deux indicateurs est positive, l'ajustement est procyclique, c'est-à-dire qu'il se fait via les créations d'emplois. Au contraire, si la corrélation est négative, l'ajustement est contra-cyclique, et passe par les destructions d'emplois (Duhautois, 2002). Ici, c'est la deuxième explication qui semble l'emporter, avec une corrélation moyenne de $-0,28$.

\section{Carte 3. Taux de réallocation et de croissance nette des emplois}

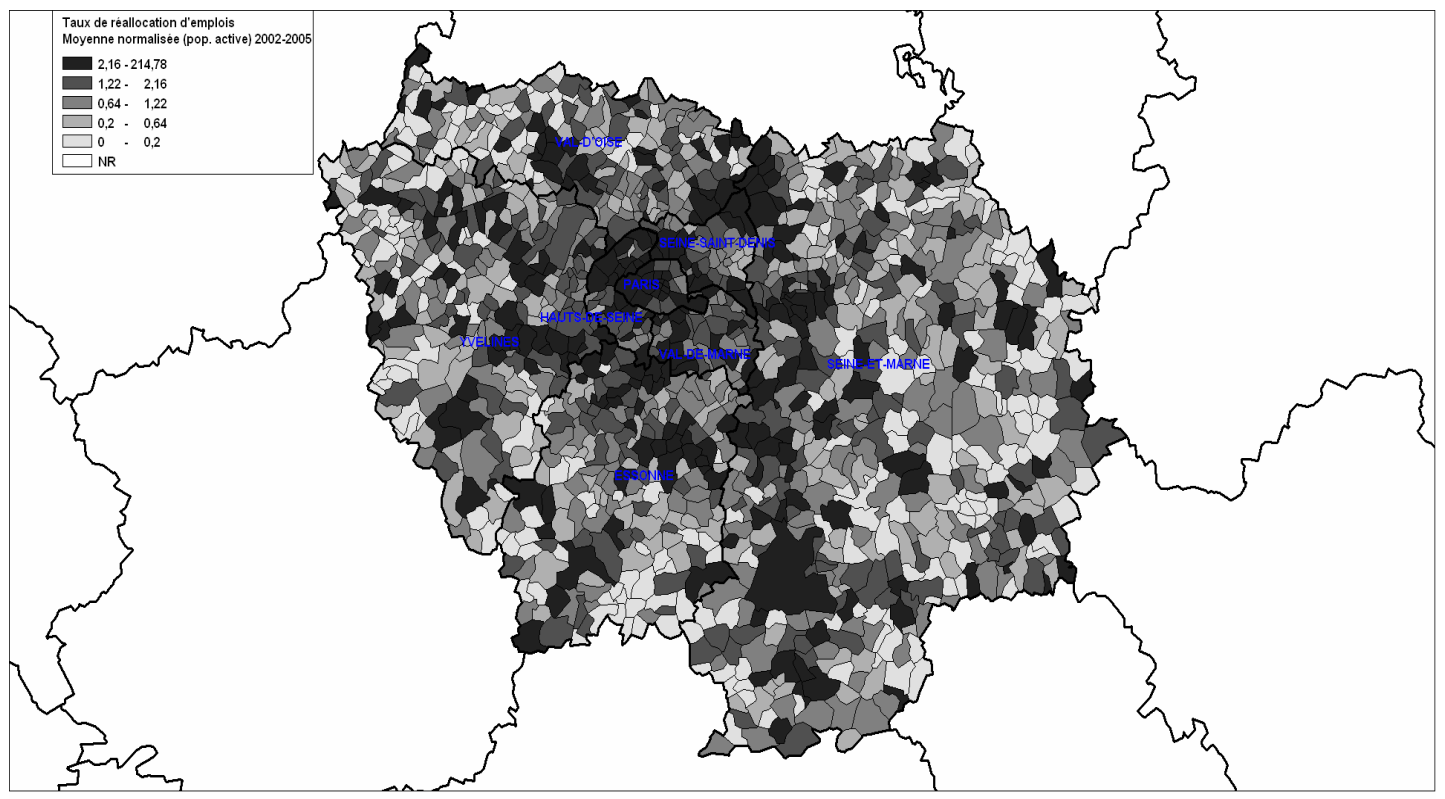

Lecture : les communes en noir sont celles où le taux de réallocation d'emplois est le plus élevé. Les communes en gris clair sont celles où le taux est le plus faible. Pour le calcul des taux, on utilise comme pondération : la population active dans la commune.

Source : DADS 2002-2005.

La cartographie du dynamisme des entreprises en termes d'emploi confirme tout d'abord la structure concentrique du bassin d'emploi ( $c f$. carte 3). En effet, c'est la région centrale de l'Île-de-France qui connaît les taux de réallocation de main-d'œuvre les plus élevés, la répartition dans l'espace des taux normalisés ${ }^{9}$ de réallocation étant comparable à celle du

\footnotetext{
${ }^{9}$ La carte 3 présente les taux de réallocation d'emplois normalisés par la taille de la population active de la commune (variable $Z_{c t}$ ), et non par l'effectif moyen des entreprises de la commune comme c'était le cas dans le
} 
nombre d'emplois par actif. Plus l'on s'éloigne du centre, plus le dynamisme est faible, ce qui corrobore l'hypothèse de mauvais appariement spatial, les chômeurs résidant loin de la région dynamique ayant plus de mal à retrouver rapidement un emploi. Cependant, s'agissant du chômage élevé de Paris et de la première couronne, cette explication ne semble pas satisfaisante. De plus, nous présentons ici des résultats qui ne tiennent pas compte de la taille des entreprises. Or les taux de création et de destruction d'emplois sont en général inversement proportionnels à la taille des entreprises (Duhautois, 2002). Il faudrait donc contrôler par exemple du secteur d'activité (qui est bien corrélé à la taille moyenne des entreprises) pour confirmer nos résultats.

\section{La mesure du mauvais appariement des compétences}

L'autre explication du chômage retenue dans la littérature théorique est l'existence d'un mauvais appariement des compétences qui s'ajoute au mauvais appariement spatial. Certains chômeurs peuvent en effet sortir difficilement du chômage non pas parce qu'il n'y a pas d'emplois près de leur lieu de résidence, mais parce qu'il n'y a pas d'emploi qui correspond à leur qualification. Il est donc intéressant de décomposer les indicateurs du dynamisme des entreprises en termes d'emploi par niveau de qualification, afin de constater la contribution de chacun de ces niveaux aux taux de création et de destruction d'emplois. En effet, les dynamiques conjoncturelles de l'emploi des ouvriers et employés non qualifiés (NQ), qualifiés (Q) et des autres niveaux de qualification (TQ) ne sont pas forcément parallèles, et au sein d'entreprises qui globalement créent des emplois, certaines détruisent pourtant des emplois NQ par exemple.

Formellement, on peut décomposer le volume des créations d'emplois en fonction des niveaux de qualification :

$$
C_{c t}=\sum_{e \in C^{+}} \Delta N_{e c t}=\sum_{e \in C^{+} \cap N Q} \Delta N_{e c t}^{N Q}+\sum_{e \in C^{+} \cap Q} \Delta N_{e c t}^{Q}+\sum_{e \in C^{+} \cap T Q} \Delta N_{e c t}^{T Q}
$$

En décomposant les trois termes du membre de droite de l'équation précédente en création et destruction brutes :

$$
C_{c t}=\sum_{e \in C^{+}} \Delta N_{e c t}=\sum_{e \in C^{+} \cap N Q^{+}} \Delta N_{e c t}^{N Q}+\sum_{e \in C^{+} \cap N Q^{-}} \Delta N_{e c t}^{N Q}+\sum_{e \in C^{+} \cap Q^{+}} \Delta N_{e c t}^{Q}+\sum_{e \in C^{+} \cap Q^{-}} \Delta N_{e c t}^{Q}+\sum_{e \in C^{+} \cap T Q^{+}} \Delta N_{e c t}^{T Q}+\sum_{e \in C^{+} \cap T Q^{-}} \Delta N_{e c t}^{T Q}
$$

De même pour les destructions brutes d'emplois :

$$
D_{c t}=\sum_{e \in C^{-}}\left|\Delta N_{e c t}\right|=\sum_{e \in C^{-} \cap N Q^{+}} \Delta N_{e c t}^{N Q}+\sum_{e \in C^{-} \cap N Q^{-}} \Delta N_{e c t}^{N Q}+\sum_{e \in C^{-} \cap Q^{+}} \Delta N_{e c t}^{Q}+\sum_{e \in C^{-} \cap Q^{-}} \Delta N_{e c t}^{Q}+\sum_{e \in C^{-} \cap T Q^{+}} \Delta N_{e c t}^{T Q}+\sum_{e \in C^{-} \cap T Q^{-}} \Delta N_{e c t}^{T Q}
$$

tableau 3, ceci afin de tenir compte des disparités importantes concernant la taille des populations d'une commune à l'autre. 
Tableau 2. Contribution des différents niveaux de qualification

\begin{tabular}{cccccccccccccc}
\hline & \multicolumn{4}{c}{ Taux de création } & \multicolumn{4}{c}{ Taux de destruction } \\
\hline & \multicolumn{3}{c}{ Taux de création } & \multicolumn{3}{c}{$\begin{array}{c}\text { Taux de } \\
\text { destruction }\end{array}$} & \multicolumn{3}{c}{ Taux de création } & \multicolumn{3}{c}{$\begin{array}{c}\text { Taux de } \\
\text { destruction }\end{array}$} \\
\hline & NQ & Q & TQ & NQ & Q & TQ & NQ & Q & TQ & NQ & Q & TQ \\
\hline $2002-2003$ & 3,9 & 3,0 & 3,6 & $-1,9$ & $-1,2$ & $-1,2$ & 1,1 & 1,0 & 1,2 & $-3,7$ & $-2,5$ & $-2,6$ \\
$2003-2004$ & 3,0 & 2,5 & 2,9 & $-1,5$ & $-1,0$ & $-1,0$ & 1,3 & 0,9 & 1,0 & $-3,7$ & $-2,8$ & $-2,7$ \\
$2004-2005$ & 3,6 & 2,8 & 2,8 & $-1,2$ & $-0,9$ & $-1,3$ & 1,2 & 0,9 & 0,8 & $-3,9$ & $-2,8$ & $-3,6$ \\
\hline
\end{tabular}

Lecture : NQ : employés et ouvriers non qualifiés ; Q : employés et ouvriers qualifiés ; TQ : autres salariés. Champ : établissements pérennes de la région Île-de-France.

Source : DADS 2002-2005.

Les résultats obtenus ( $c f$. tableau 2) témoignent surtout d'un ajustement par les non qualifiés, que ce soit au niveau des créations, comme au niveau des destructions, puisque dans tous les cas, ils ont les contributions les plus élevées. Cela traduit peut-être une fragilité plus grande de cette catégorie sur le marché du travail, davantage soumise aux aléas conjoncturels, ainsi que l'importance de l'emploi NQ en Île-de-France où le secteur des services est très présent (Davezies, 2007). L'emploi TQ semble être plus dynamique en termes de créations d'emplois que l'emploi $\mathrm{Q}$, dans une région où la population est en moyenne plus qualifiée que sur l'ensemble du territoire (Buisson et Mignot, 2005).

D'un point de vue géographique, la comparaison entre la cartographie des sans diplôme avec celle de la contribution de l'emploi non qualifié à la dynamique locale de l'emploi ( $c f$. carte 4) suggère que l'éloignement des chômeurs à faible niveau de qualification des emplois qui leur correspondent est une explication plausible de leur difficulté à retrouver un emploi. On constate ainsi par exemple que l'est des Yvelines, le nord de l'Essonne, les Hauts-de-Seine ou encore le sud et l'est de Paris sont des zones où l'emploi non qualifié est très dynamique, alors qu'une large majorité des travailleurs qui y résident sont diplômés. Au contraire, l'est de la Seine-et-Marne comporte de nombreuses communes peu dynamiques et une forte proportion de travailleurs non qualifiés. Le mésappariement serait donc ici géographique, mais causé par l'inadéquation des compétences des travailleurs aux emplois proposés sur place.

Pour mesurer le mésappariement des compétences, nous adaptons l'indicateur proposé par Layard et alii. $(1990)^{10}$, qui mesure l'écart entre les proportions relatives de chômeurs par qualification selon les communes. Formellement, il correspond à la demi-variance du rapport entre le taux de chômage par qualification $u_{q}$ (ouvriers, employés, professions intermédiaires et cadres) et le taux de chômage total de la commune $u$ :

$$
I_{L J S}=\frac{1}{2} \mathrm{~V}\left(\frac{u_{q}}{u}\right) \text { avec } 0<I_{L J S}<1
$$

Nous nous intéressons en particulier au mésappariement des compétences susceptible de toucher les non qualifiés. Nous le mesurons comme l'écart pour chaque commune entre la

\footnotetext{
${ }^{10}$ On a également testé l'indicateur de Sneessens et Shadman-Mehta (1995) qui mesure le décalage entre les structures de l'offre et de la demande en comparant la proportion d'emplois qualifiés dans l'emploi total et la proportion de travailleurs qualifiés dans la population active ; et les résultats obtenus sont comparables.
} 
proportion des non diplômés qui y résident et le dynamisme local de l'emploi non qualifié ${ }^{11}$, qui semblait potentiellement grand à la lecture de la carte 4 .

${ }^{11}$ Il s'agit ici d'un indicateur totalement empirique, contrairement à $I_{L J S}$, qui souffre de plus d'être construit à partir de données ne s'appuyant pas tout à fait sur les mêmes nomenclatures (diplôme pour le recensement 1999, qualifications pour les DADS 2002-2005). 


\section{Carte 4. Proportion de sans diplôme et contribution de l'emploi non qualifié}

au taux de création d'emplois

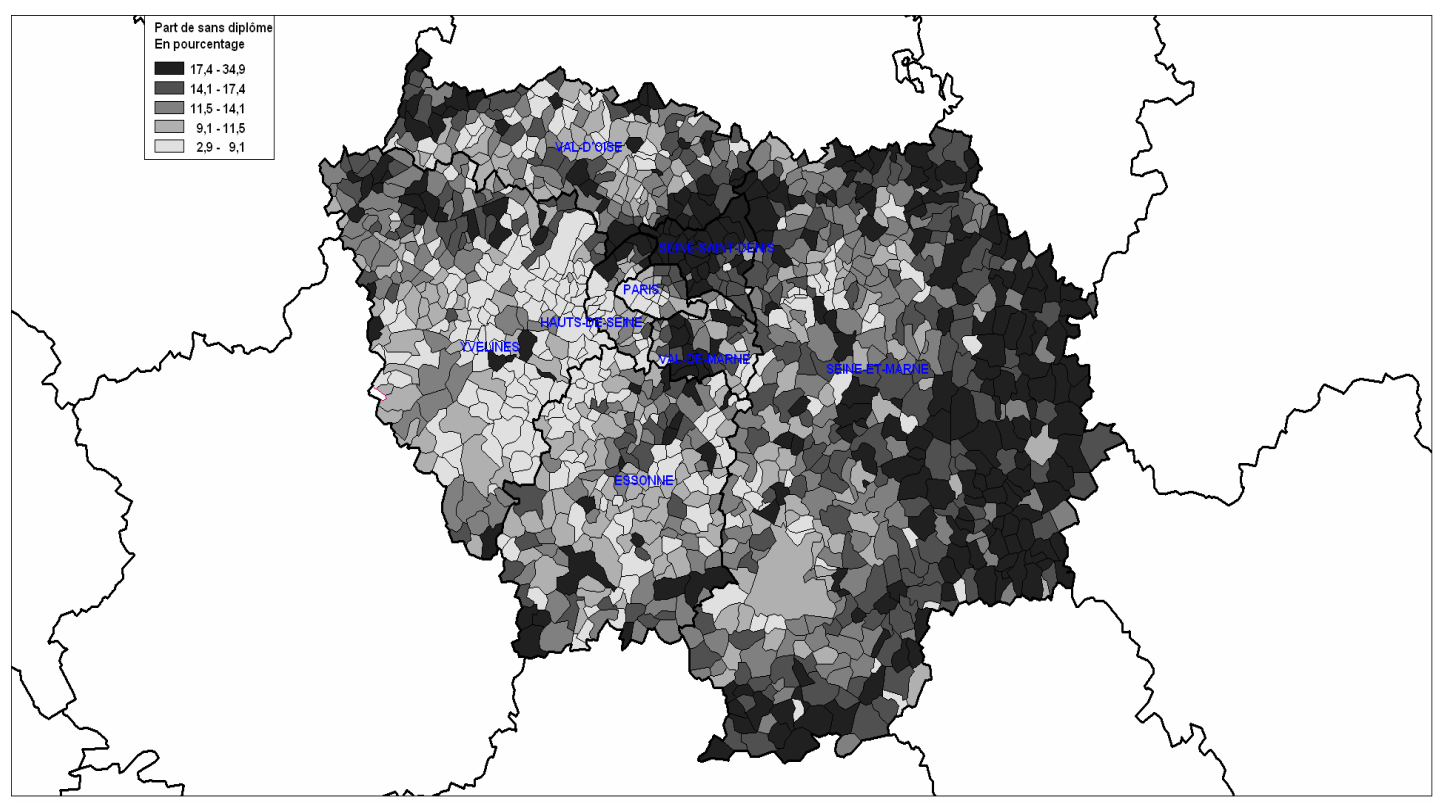

Lecture : Les communes en noir sont celles ou la part des individus sans diplôme est la plus importante. Les communes en gris clair sont celles ou la part est la plus faible.

Source : Recensement 1999 de l'Insee.

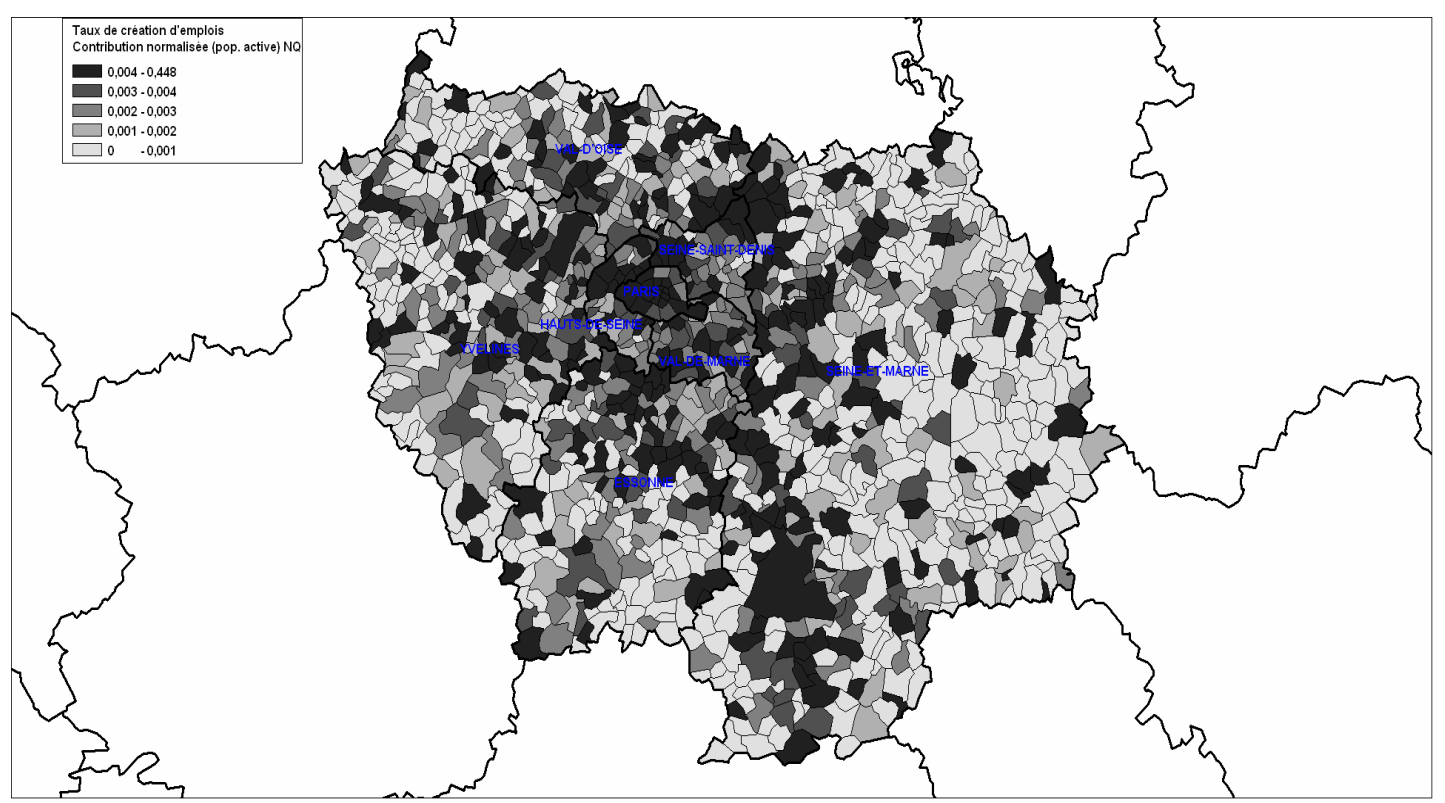

Lecture : les communes en noir sont celles où la contribution de l'emploi non qualifié à la dynamique locale de l'emploi est la plus forte. Les communes en gris clair sont celles où cette contribution est la plus faible. Pour le calcul des taux, on utilise comme pondération: la population active dans la commune.

Source : DADS 2002-2005. 


\section{Ségrégation et effets de pairs}

Pour contrôler des éventuels effets de ségrégation, nous avons construit une typologie des communes d'île-de-France à partir d'une analyse en composantes principales puis d'une classification ascendante hiérarchique (critère de Ward) prenant appui sur des variables mesurant la proportion de chaque CSP, la répartition relative des diplômes, ainsi que la proportion de familles monoparentales et d'étrangers résidant dans chaque commune. Cette classification permet de former quatre groupes de communes relativement homogènes du point de vue de leur population : communes à population majoritairement très qualifiée, où résident essentiellement des cadres (type I), communes à population qualifiée avec une proportion de familles monoparentales et d'étrangers supérieure à la moyenne (type II), communes où les résidents sont majoritairement ouvriers et peu diplômés (type III), et communes où la proportion d'ouvriers, de familles monoparentales et d'étrangers est élevée (type IV) ( $c f$. carte 5). Cette classification ad hoc souffre néanmoins de l'absence de fondements théoriques solides permettant de sélectionner les variables pertinentes concernant la construction d'effets de ségrégation. Cette limite est néanmoins acceptable dans l'optique qui est la nôtre, à savoir tester l'existence d'un mauvais appariement spatial, puisque les éventuels effets de pairs ne nous servent ici que de variables de contrôle. Or notre typologie présente l'avantage de résumer synthétiquement l'information disponible sur les caractéristiques sociodémographiques des résidents d'île-de-France. D'autre part, la proportion de foyers imposables à l'IRPP permet de contrôler des différences de revenus moyens entre les communes.

\section{Carte 5. Classification ascendante hiérarchique des communes}

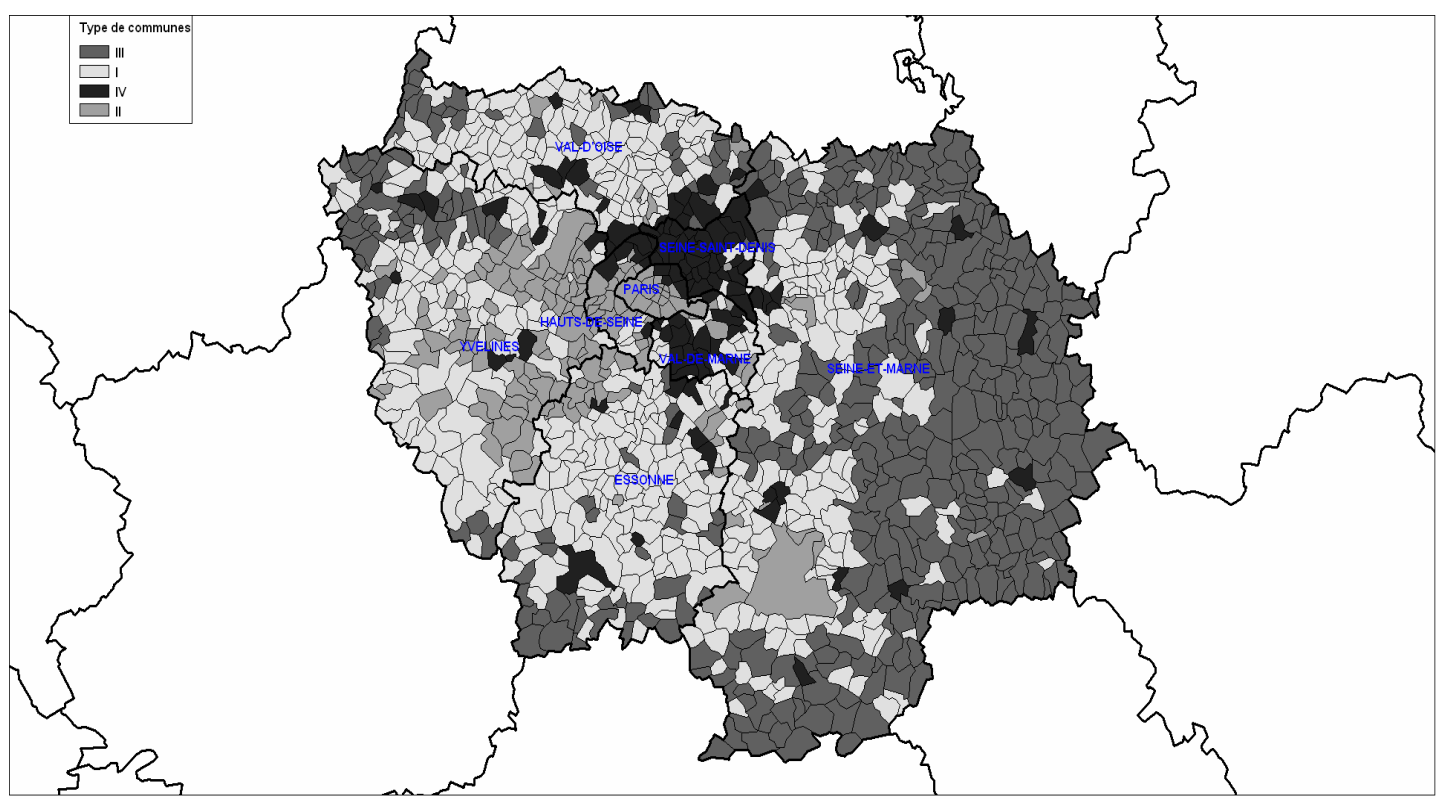

Lecture : Chaque couleur représente un type de communes identifié par la typologie (CAH). Source : Recensement 1999 de l'Insee.

\section{Logement et statut résidentiel des ménages}

Le logement peut également être un élément explicatif des durées de chômage observées. Pour le prendre en compte, nous ajoutons des variables relatives au statut résidentiel des ménages de la commune. Nous distinguons les propriétaires (qui comprennent également les accédants à la propriété), les locataires du secteur privé et les locataires du secteur public (locataires de logement sociaux de type HLM ou autres). Nous créons ainsi des variables qui 
renseignent sur la part des propriétaires de la commune, la part des locataires privés et la part des locataires publics. Introduire ces différents statuts résidentiels dans une régression, nous permet de tester la fameuse hypothèse d'Oswald $(1996,1999)$. Selon cette hypothèse, des liens peuvent exister entre le marché du logement et le marché du travail ${ }^{12}$. Elle s'appuie sur le fait selon lequel les propriétaires seraient moins mobiles que les locataires. L'augmentation du nombre de propriétaires et d'accédants à la propriété aurait pour conséquence de diminuer les appariements entre les demandeurs d'emplois et les postes vacants. Dans le cas français, les travaux de Brunet et Lesueur (2003), Brunet et alii. (2007) ou Brunet et Lesueur (2009) montrent, entre autres, une influence du statut résidentiel sur la durée de chômage et particulièrement du statut de propriétaire. Les auteurs confirment en partie l'hypothèse d'Oswald, en montrant que le fait d'être propriétaire peut augmenter la durée de chômage. C'est ce type de lien que l'on se propose de vérifier lorsque l'on introduit ces variables explicatives.

\section{Stratégie pour une estimation en économétrie spatiale}

\section{L'auto-corrélation spatiale}

Dans la mesure où nos données sont spatialisées (on connaît la distribution des variables par commune de l'Île-de-France), nous devons mettre en œuvre des modèles économétriques adaptés, qui tiennent compte d'une possible auto-corrélation spatiale et exploitent l'information supplémentaire dont nous disposons, à savoir la répartition géographique du phénomène étudié. La modélisation doit ainsi tenir compte de la structure spatiale particulière de la durée du chômage et du dynamisme de l'emploi, puisque les deux phénomènes ne semblent pas aléatoirement répartis sur le territoire, mais sont au contraire fortement contrastés en fonction des zones étudiées, certaines communes voisines ayant des profils proches quand d'autres s'opposent nettement. L'introduction d'un critère géographique permet ainsi de tenir compte d'un double phénomène : d'une part, la possible dépendance dans l'espace de plusieurs variables entre elles (qui peuvent par exemple varier de la même manière en fonction du lieu d'observation) ou des valeurs prises par une même variable (dont la valeur en un endroit dépend de la valeur voisine); d'autre part, la potentielle nonstationnarité dans l'espace de la relation entre plusieurs variables (l'influence d'une variable sur une autre pouvant varier en fonction de la localisation).

Concernant la durée du chômage qui constitue notre variable d'intérêt, on s'intéresse à son auto-corrélation spatiale, c'est-à-dire à la dépendance dans l'espace entre les différentes valeurs prises par la variable. Une auto-corrélation spatiale positive traduit la coïncidence de valeurs similaires en des endroits similaires, alors que l'auto-corrélation spatiale négative est la manifestation de valeurs très différentes dans des endroits proches. Dans les deux cas, la répartition géographique de la variable n'est pas aléatoire et il faut en tenir compte dans la modélisation. Dans le cas qui nous intéresse, les sources de l'auto-corrélation spatiale peuvent être un mauvais appariement spatial entre les travailleurs et les emplois, mais aussi des effets de ségrégation (de pairs), c'est-à-dire l'idée selon laquelle la composition d'un quartier ou d'une commune influe sur le retour à l'emploi. Il serait ainsi plus difficile de sortir du chômage lorsque le voisinage est lui-même faiblement actif, notamment parce que dans ce cas les réseaux sociaux entretenus pas les individus sont faiblement favorables à la reprise

\footnotetext{
${ }^{12}$ L'auteur avance qu'un pourcentage de propriétaires élevé dans un pays ou dans une région est responsable d'un important niveau de chômage.
} 
d'emploi (Selod et Zenou, 2006). Il faudra donc contrôler de la composition de la population des communes pour mesurer le mauvais appariement spatial.

Afin d'avoir une confirmation de la présence d'auto-corrélation spatiale, nous calculons le coefficient d'auto-corrélation I de Moran pour les durées de chômage. Le I de Moran peut être interprété comme le rapport de la covariance entre observations contiguës et de la variance totale observée de l'échantillon (Jayet, 1993). Il est donné par :

$$
I_{M}=\frac{n}{\sum_{i} \sum_{j} w_{i j}} \frac{\sum_{i} \sum_{j} w_{i j}\left(x_{i}-\bar{x}\right)\left(x_{j}-\bar{x}\right)}{\sum_{i}\left(x_{i}-\bar{x}\right)^{2}}
$$

où $w_{i j}$ est un poids qui permet de rendre compte de la proximité géographique des unités spatiales $i$ et $j$.

Par ailleurs, lorsque $\mathrm{I}>\mathrm{E}[\mathrm{I}]=(\mathrm{n}-1)^{-1} \mathrm{I}$ (respectivement $\mathrm{I}<\mathrm{E}[\mathrm{I}]$ ), les valeurs prises par les durées ne sont pas disposées aléatoirement mais sont proches (respectivement éloignées) pour deux unités spatiales voisines. Les unités spatiales géographiquement proches sont aussi statistiquement proches (respectivement éloignées) et nous concluons à la présence d'une auto-corrélation spatiale positive (respectivement négative). Lorsque I est proche de $\mathrm{E}[\mathrm{I}]$, nous concluons à l'absence d'auto-corrélation spatiale. Dans ce cas, nous ne pouvons établir aucun lien entre la proximité statistique et la proximité géographique des unités spatiales.

Dans les faits, le calcul de l'indice I de Moran est sensible à la définition de la matrice de poids spatiaux $\mathrm{W}\left(w_{i j}\right)$. Il existe effectivement plusieurs critères pour déterminer les unités spatiales qui seront considérées comme voisines : contiguïté (déplacements de type reine, fou ou roi), plus proches voisins, distance. Au vu des cartes précédemment analysées des durées de chômage, et l'existence de grappes de communes homogènes, on choisit de construire une matrice de contiguïté, où les communes ont des liens avec tous leurs voisins immédiats. Afin de couvrir la superficie des zones homogènes de communes, nous autorisons l'interaction jusqu'à trois communes voisines consécutives. Selon le vocabulaire consacré emprunté aux échecs, on sélectionne donc un critère Reine à l'ordre 3 (déplacement de la reine dans toutes les cases mitoyennes : on considère que les communes voisines interagissent jusqu'à franchir trois frontières administratives consécutives). Nous présentons toutefois, dans le tableau suivant, les coefficients d'auto-corrélation (I de Moran) du taux de sortie de chômage et du taux de chômage obtenus pour différents types de matrices de poids spatiaux.

Tableau 3. Auto-corrélation spatiale globale de la durée de chômage

\begin{tabular}{lccc}
\hline Matrice W & I de Moran & Ecart-type & p-valeur \\
\hline Reine 1 & 0,648 & 0,020 & 0,001 \\
Reine 2 & 0,485 & 0,013 & 0,001 \\
Reine 3 & 0,379 & 0,011 & 0,001 \\
Distance $<5 \mathrm{~km}$ & 0,601 & 0,016 & 0,001 \\
\cline { 2 - 4 } Source & FHS de Pôle Emploi 2006 et recensement de la population (1999). \\
Notes : E[I] = -0,0009.
\end{tabular}

Quel que soit le type de matrice retenu, nous constatons que la durée de chômage présente une auto-corrélation spatiale significative positive relativement élevée. Deux communes éloignées géographiquement ont donc des durées de chômage très différentes. Des communes 
voisines géographiquement sont aussi des communes voisines en termes de durées de chômage. Pour considérer ce problème d'auto-corrélation spatiale dans nos données, il importe de mettre en place le modèle adéquat.

\section{Spécification}

Comme point de départ, nous considérons le modèle suivant pour expliquer la durée de chômage dans les communes franciliennes pour lesquelles elle a pu être calculée :

$$
Y_{i}=\beta \operatorname{Emp}_{i}+\gamma S p_{i}+\delta S k_{i}+\eta \operatorname{Segreg}_{i}+v \log _{i}+\phi \operatorname{Pol}_{i}+\varepsilon_{i}
$$

où $Y_{i}$ est la durée de chômage pour une commune $i$ donnée. Emp regroupe les indicateurs relatifs au dynamisme de l'emploi local (taux de création et taux de destruction). $S p$ est un vecteur de variables mesurant l'accessibilité aux emplois pour chacune des communes de la région. Outre la distance moyenne domicile-travail, nous retenons la densité d'emplois dans un rayon de 20 kilomètres. $S k$ est un vecteur de variables relatives à l'inadéquation locale entre les compétences des individus et celles requises pour les emplois. Il inclut l'indice de Layard, Jackman et Savouri (LJS) et l'écart entre proportion de sans diplôme et taux de création d'emplois non qualifiés. Le vecteur Segreg regroupe des indicatrices issues de la classification ascendante hiérarchique. La commune affiche la valeur 1 si elle appartient à une classe donnée, 0 sinon. Il comprend également la part de foyers imposables à l'Impôt sur le Revenu des Personnes Physiques (IRPP). Enfin, Log inclut des variables relatives au statut résidentiel des ménages (part des propriétaires, part des locataires HLM et part des locataires privés), tandis que $\mathrm{Pol}$ inclut des variables relatives aux politiques locales d'emploi (proportion de salariés en emploi aidé).

Une modélisation toutes choses égales par ailleurs permet de tester les phénomènes de mauvais appariement spatial entre l'offre et la demande de travail. Cependant, en raison de l'auto-corrélation spatiale, on ne peut plus mettre en œuvre la méthode standard des Moindres Carrés Ordinaires, car la covariance entre les observations n'est plus nulle. Il faut alors choisir parmi les modèles classiques de l'économétrie spatiale celui qui correspond le mieux à notre problème. Selon la littérature en économétrie spatiale, deux modèles traditionnels peuvent être spécifiés pour prendre en compte l'auto-corrélation (LeSage, 1998 ; Anselin, 2006 ; Le Gallo, 2002) : un modèle SAR dans lequel la variable dépendante suit un processus spatialement autoregressif. Il permet de modéliser les effets de débordement en intégrant une variable endogène spatialement décalée $(\mathrm{W} y)$ en plus de la matrice des variables indépendantes ; un modèle SEM dans lequel la dépendance spatiale se rattache aux erreurs.

Le modèle SAR peut s'écrire de la façon suivante :

$$
Y_{i}=\rho W Y_{i}+\beta E m p_{i}+\gamma S p_{i}+\delta S k_{i}+\eta \text { Segreg }_{i}+v \log _{i}+\phi \operatorname{Pol}_{i}+\varepsilon_{i} \quad \mathcal{E} \sim \mathrm{N}\left(0, \sigma^{2} I\right)
$$

où le paramètre estimé $\rho$ mesure la dépendance spatiale des observations.

Le modèle SEM s'écrit, lui, de la façon suivante :

$$
\begin{gathered}
Y_{i}=\beta E m p_{i}+\gamma S p_{i}+\delta S k_{i}+\eta \text { Segreg }_{i}+\nu \log _{i}+\phi \text { Pol }_{i}+\varepsilon_{i} \\
\text { avec } \varepsilon=\lambda W \varepsilon+u \text { et } u \sim \mathrm{N}\left(0, \sigma^{2} I\right)
\end{gathered}
$$


où $\lambda$ est le paramètre représentant l'intensité de la dépendance spatiale entre les résidus de la régression. Cette dépendance est qualifiée de dépendance spatiale de nuisance.

A priori, les deux modèles semblent applicables. Dans le modèle SAR, l'auto-corrélation spatiale des observations est capturée par la variable endogène spatialement décalée $W Y$ et reflète l'idée selon laquelle le taux de sortie du chômage dans une commune est influencé par celui des communes voisines. Dans le modèle SEM, nous considérons la dépendance spatiale comme une nuisance statistique qui peut s'expliquer par des problèmes de mauvaises spécifications (variables omises, mauvaise échelle géographique retenue etc.). Lorsqu'une variable endogène décalée est ignorée dans la spécification du modèle, alors qu'elle est présente dans le processus générateur des données, les estimateurs des MCO dans le modèle représenté par l'équation (1) seront biaisés et non convergents (contrairement à l'équation (2)). De même, ne pas considérer la dépendance spatiale au niveau des erreurs et estimer l'équation (2), plutôt que l'équation (3), produit des estimateurs biaisés et inconsistants. Quel que soit le modèle spatial retenu, les paramètres de l'équation sont estimés par la méthode du Maximum de Vraisemblance.

Dans la pratique, il existe des tests qui permettent de trancher entre les deux formes de dépendance spatiale. Il s'agit des tests du multiplicateur de Lagrange (LMERR, LMLAG et leurs versions robustes R-LMERR et R-LMLAG). Toutefois, le contexte de l'analyse et la structure des données peuvent permettre de s'affranchir de ces tests et de recourir à la méthode la plus logiquement appropriée.

Dans notre cas, nous supposons que l'auto-corrélation spatiale provient de l'échelle d'analyse qui est retenue. En effet, la façon dont les données spatiales sont agrégées peut avoir un effet sur la mesure de l'auto-corrélation spatiale. Il s'agit du "Modifiable Areal Unit Problem » (MAUP) (Le Gallo, 2002). Celui-ci regroupe deux problèmes potentiels : (i) l'auto-corrélation spatiale peut être affectée par le niveau d'agrégation utilisé. On parle d'effet d'échelle. (ii) La façon de découper une zone en plusieurs subdivisions donne lieu à de nombreuses configurations spatiales. L'auto-corrélation peut être liée à ce problème de forme des unités spatiales. Or, ce sont deux problèmes que l'on retrouve potentiellement dans notre analyse à l'échelle de la commune ${ }^{13}$. Dans ce cas, le modèle approprié est le modèle SEM.

Si l'auto-corrélation spatiale provient du fait que les données sont affectées par des processus qui relient des lieux différents et qui sont à l'origine d'une organisation particulière des activités dans l'espace, il est d'usage de recourir au modèle SAR. Dit autrement, l'organisation et la structure spatiales inhérentes aux phénomènes économiques et sociaux produisent des interactions complexes qui doivent être intégrées dans la modélisation des phénomènes spatiaux. Ces interactions spatiales peuvent prendre la forme d'effets de diffusion et d'effets de débordement, qui supposent que les observations s'influencent. Néanmoins, ici, il est difficile d'envisager que le chômage dans une commune puisse influer le chômage dans les communes voisines. Si des effets de pairs peuvent exister, ils jouent davantage à l'échelle individuelle ou à l'échelle du quartier plutôt que de la commune ${ }^{14}$.

\footnotetext{
${ }^{13}$ En d'autres termes, nous supposons que l'échelle utilisée lors de la collecte de données (communale) est agrégée, et peut ne pas effectivement correspondre à l'échelle à laquelle le processus que l'on étudie joue. Ceci donne lieu à des erreurs de mesures.

${ }^{14}$ Les nombreux travaux en économie urbaine qui s'intéressent aux liens entre effets de pairs et performances sur le marché du travail s'appuient généralement sur des analyses à l'échelle du quartier. Cela se justifie par le fait que c'est à cette échelle que les individus sont susceptibles d'intéragir entre eux (voir les revues de littérature de Dietz, 2002 ou Galster, 2008).
} 
Pour ces différentes raisons, nous avons recours à un modèle SEM. Puisque nous raisonnons toujours à l'échelle de la commune et que nous cherchons à expliquer les disparités de chômage, le raisonnent décrit est valable dans toutes les configurations qui seront présentées. Nous estimons donc l'équation (3) par Maximum de Vraisemblance. Nous retenons une matrice de contiguïté de type Reine à l'ordre 3 pour définir le voisinage.

Le recours à ces régressions agrégées permet de mettre en évidence des relations spatiales contribuant à la formation du chômage au niveau des communes. Le recours aux "taux nets" permet de contrôler d'éventuels effets de composition (par exemple, une commune comportant une proportion élevée d'étrangers connaît un taux de chômage élevé car les étrangers sont, toutes choses égales par ailleurs, plus au chômage) puisque nous raisonnons comme si chaque commune avait la composition moyenne de la région. Dans ces conditions, notre estimation permet de prendre la pleine mesure des effets de ségrégation (une commune comportant une proportion élevée d'étrangers connaît un taux de chômage élevé pour des raisons de stigmatisation du quartier par les employeurs ou encore à cause de la faible qualité des réseaux sociaux). Cependant, cette méthode est sujette au risque de l' "erreur écologique », une erreur d'interprétation qui consiste à inférer à un niveau individuel des résultats obtenus à un niveau agrégé (Marpsat, 1999).

De plus, mettre en relation les résultats des individus à leur localisation résidentielle pose le problème de l'endogénéité des choix de localisations. C'est typiquement le cas si l'on part du postulat que les individus ne choisissent pas leur lieu de résidence au hasard. Il est probable que des individus se regroupent spatialement en fonction de caractéristiques similaires et de résultats identiques sur le marché du travail. Dans ces conditions, il est difficile de distinguer si un individu est au chômage parce qu'il vit dans un quartier particulier ou bien si cet individu vit dans ce quartier parce qu'il est au chômage. Dans nos estimations, nous ne prenons pas en compte ce biais.

\section{Résultats des estimations}

Les résultats de nos estimations sont présentés dans le tableau 4. Le modèle (1) correspond au test des effets des variations de la demande de travail sur la sortie du chômage, que nous conservons systématiquement comme variable explicative en raison du poids théorique que nous leur avons donné antérieurement. Le modèle (2) teste l'existence d'un mauvais appariement spatial, alors que le modèle (3) interroge l'existence d'un mauvais appariement des compétences. Le modèle (4) introduit le contrôle des effets de pairs, le modèle (5) celui des indicatrices par département, et le modèle (6) le logement et la politique locale de l'emploi. Nos modèles expliquent entre un tiers et $40 \%$ de la variance de la durée nette de chômage.

Concernant le dynamisme local de l'emploi, les résultats sont conformes aux attentes ${ }^{15}$ : lorsque le taux de création d'emplois sur le territoire de la commune est élevé, les chômeurs retrouvent plus rapidement un emploi, ce qui souligne la pertinence de l'analyse en termes d'éloignement géographique des chômeurs aux emplois. Il en va de même pour l'effet du taux

\footnotetext{
${ }^{15}$ Le test de Breush-Pagan suggère l'existence d'hétéroscédasticité dans le modèle (1), cependant il n'est plus significatif dans l'ensemble des autres spécifications, on n'en tient donc finalement pas compte.
} 
de destruction, qui semble favoriser un chômage local de longue durée, bien que le coefficient soit globalement plus faible et moins significatif que pour les créations. Les effets locaux de la demande de travail sont donc avérés : plus que les seules caractéristiques des chômeurs dont l'effet est ici neutralisé - c'est le dynamisme des entreprises créatrices d'emplois qui offre des opportunités aux chômeurs de se réinsérer sur le marché du travail, ce qui plaide pour un renforcement des politiques locales de demande. L'impact négatif de la proportion d'emplois aidés dans la commune sur la durée de chômage va également dans ce sens : la création d'emplois protégés permet aux chômeurs de retrouver rapidement une activité proche du marché du travail «standard», mesures actuellement considérées comme relativement efficaces ${ }^{16}$.

L'interprétation des résultats obtenus avec les variables de mauvais appariement spatial s'avère plus délicate. En effet, si la distance entre le domicile et le lieu de travail semble bien jouer en défaveur du retour à l'emploi ainsi qu'on l'attendait, la variable de concentration des emplois à proximité semble au contraire défavoriser le retour à l'emploi des chômeurs qui résident dans cette zone. L'effet positif de la proximité aux emplois induit par le mauvais appariement spatial pourrait ainsi être modéré par un effet de concurrence locale entre les actifs (actifs occupés et chômeurs) dans des zones où la densité d'emplois est élevée. Cependant, cet effet n'est pas toujours significatif, et la robustesse de ces deux variables varie avec l'introduction de variables de contrôle supplémentaires. Par conséquent, il est assez difficile de trancher sur les effets potentiels du problème de mauvais appariement spatial. S'il est probable que ce phénomène soit déterminant pour les communes les plus en marge de la région (Seine et Marne, Val d'Oise, Yvelines), on peut supposer qu'il n'explique pas les durées de chômage élevées observées à Paris et dans un certain nombre de communes limitrophes.

Lorsqu'on introduit l'indice de Layard, Jackman et Savouri dans la régression, on constate que l'effet du mauvais appariement des compétences est potentiellement très élevé en Île-deFrance. Cependant, ce mésappariement mesuré sur l'ensemble des niveaux de qualification est absorbé par l'effet du mauvais appariement spatial pour les non qualifiés, qui semble être particulièrement fort, les chômeurs sans diplôme résidant majoritairement dans des communes où l'emploi non qualifié est faiblement dynamique. Le phénomène «brut» de mauvais appariement spatial, qui traduit la distance géographique entre les emplois et les chômeurs, se double donc d'un effet lié à la distance entre des emplois accessibles par type de qualification. L'idée d'un renforcement mutuel entre les deux types de mésappariement est donc ici corroborée, les résultats incitant à penser que le mauvais appariement spatial ne joue pas de la même façon pour toutes les catégories de main-d'œuvre.

Les effets de pairs semblent jouer eux aussi un rôle non négligeable, puisque le type de commune dans laquelle résident les chômeurs influence la rapidité de sortie du chômage. Cependant, il est difficile de dire si ce sont les caractéristiques en propre des travailleurs qui jouent (par exemple le fait d'être cadre) ou davantage les externalités dues à la composition du voisinage (le fait d'être un cadre qui habite dans une commune où réside une majorité de cadres, voire même le simple fait d'avoir des voisins cadres quelle que soit sa propre qualification). En effet se pose ici le problème d'endogénéité qui interdit de trancher sur le sens de la causalité : certaines communes connaissent des durées moyennes de chômage plus courtes que d'autres, mais est-ce parce qu'un certain type de population y est majoritaire, ou bien cette population ne vient-elle pas s'installer dans cette commune car les opportunités

${ }^{16} C f$. par exemple Forslund et alii. (2004) sur données suédoises. 
d'insertion sur le marché du travail y sont meilleures ex ante ? On peut ainsi concevoir un processus auto-entretenu où ce sont les mieux «armés » sur le marché du travail qui viennent s'installer dans les zones d'emploi favorables, renforçant de fait l'attrait de ces communes. Au contraire, les territoires où le retour à l'emploi s'avère difficile seraient abandonnés par les travailleurs les mieux insérés, créant ex post des effets de pairs pour les travailleurs contraints d'y demeurer.

Enfin, lorsque l'on s'intéresse au rôle du logement, nous observons que le statut résidentiel des ménages dans la commune a une influence directe sur la durée de chômage observée. Concrètement, lorsque nous introduisons la part des locataires privés comme référence nous observons que la part des propriétaires, mais aussi la part des locataires publics augmente la durée de chômage. Le résultat peut paraître surprenant car ces deux caractéristiques semblent refléter des réalités très différentes. Les locataires de logements publics incluent pour une large part des locataires HLM, or ces derniers ont bien souvent des caractéristiques jugées plus « défavorables » sur le marché du travail que les seuls propriétaires.

L'effet plus élevé de la part de locataires publics peut être dû à quelques contraintes ou incitations qui rendent ces locataires moins mobiles que les propriétaires. En effet, en France, les familles avec un revenu en dessous d'un seuil donné et avec certaines caractéristiques démographiques (par exemple les familles nombreuses, les familles mono-parentales etc.) peuvent demander des logements à loyers modérés (c'est-à-dire les logements sociaux). Comme le seuil est élevé, le nombre de familles pouvant potentiellement prétendre à ce type de logement est élevé et cela peut conduire à une attente considérable. Quand les familles obtiennent leur logement, elles ont le droit d'y rester pour une période indéterminée et ce, quel que soit leurs revenus. En outre, le loyer n'est que faiblement indexé au revenu du ménage et reste globalement plus faible que dans le secteur privé. Pour ces différentes raisons, les individus ont très peu d'incitations à quitter leur logement et ils font donc face au même type de dilemmes que les propriétaires durant leur recherche d'emploi. En résumé, nous pouvons supposer que locataires privés sont plus mobiles que les propriétaires et les propriétaires sont vraisemblablement plus mobiles que les locataires publics. L'effet de chacun des statuts résidentiels sur le chômage est progressif et lié à leur degré de mobilité ${ }^{17}$. Cet effet peut se justifier par le fait que les locataires privés peuvent plus facilement changer de localisation et accroitre leurs chances de trouver un emploi que les autres statuts résidentiels. Les résultats du modèle semblent donc être en adéquation avec l'hypothèse avancée par Oswald qui montre que la moindre mobilité des individus est un frein à la recherche d'emploi.

\footnotetext{
${ }^{17}$ Cette distinction entre statuts résidentiels a déjà été retenue dans un travail de Brunet et alii. (2007). Les auteurs ont également mis en évidence des différences sur le taux de sortie du chômage entre propriétaires et locataires (privés ou publics). Leurs résultats sur données françaises vont à l'encontre des nôtres : ils montrent que le statut de propriétaire augmente le taux de sortie du chômage, tandis que le statut de locataire le diminue. Toutefois, il est assez difficile de comparer des résultats de travaux sur données agrégées avec ceux sur données individuelles, en raison du risque de l' «erreur écologique » (Marpsat, 1999). C'est le type d'erreur qui peut se produire dès lors que l'on infère à un niveau individuel des résultats obtenus à un niveau agrégé.
} 
Tableau 4. Déterminants de la durée locale du chômage (modèle SEM)

\begin{tabular}{|c|c|c|c|c|c|c|}
\hline & (1) & $(2)$ & (3) & (4) & (5) & (6) \\
\hline$\lambda$ & $0,81 * * *(0,04)$ & $0,81 * * *(0,04)$ & $0,79 * * *(0,04)$ & $0,80 * * *(0,04)$ & $0,19 * *(0,08)$ & $0,88 * * *(0,03)$ \\
\hline Constante & $10,71 * * *(0,18)$ & $10,39 * * *(0,32)$ & $10,32 * * *(0,18)$ & $10,34 * * *(0,36)$ & $8,89 * * *(037)$ & $10,09 * * *(0,59)$ \\
\hline \multicolumn{7}{|l|}{ Dynamisme local de l'emploi } \\
\hline Taux de création & $-0,07 * *(0,03)$ & $-0,06 * *(0,03)$ & $-0,06 * *(0,03)$ & $-0,06 * *(0,03)$ & $-0,04 *(0,02)$ & $-0,06 * *(0,03)$ \\
\hline Taux de destruction & $0,04 *(0,02)$ & $0,04 *(0,02)$ & $0,04 *(0,02)$ & $0,04 *(0,02)$ & $0,04 *(0,02)$ & $0,04 *(0,02)$ \\
\hline \multicolumn{7}{|l|}{ Mauvais appariement spatial } \\
\hline Distance domicile-travail & & $0,02 * *(0,01)$ & & $0,01(0,01)$ & $0,04 * * *(0.01)$ & $0,003(0,01)$ \\
\hline Densité d'emploi à 20 km & & $0,28 *(0.16)$ & & $0,19(0,19)$ & $0,37 * *(0,18)$ & $0,32 *(0,18)$ \\
\hline \multicolumn{7}{|l|}{ Mauvais appariement des compétences } \\
\hline Indice de LJS & & & $2,62(2,51)$ & $2,49(2,51)$ & $4,88 * *(2,59)$ & $5,86 * *(2,44)$ \\
\hline $\begin{array}{l}\text { Ecart entre proportion de sans diplôme et } \\
\text { taux de création d'emplois non qualifiés }\end{array}$ & & & $2,62 * * *(0,68)$ & $1,70 * * *(0,84)$ & $3,47 * * *(0,84)$ & $1,75 * *(0,86)$ \\
\hline \multicolumn{7}{|l|}{ Effets de ségrégation } \\
\hline Communes de type I & & & & $-0,35 * * *(0,13)$ & $-0,22 * *(0,13)$ & $-0,34 * *(0,13)$ \\
\hline Communes de type II & & & & $-0,32 * *(0,16)$ & $-0,15(0,16)$ & $-0,26 *(0,16)$ \\
\hline Communes de type III & & & & $-0,39 * * *(0,14)$ & $-0,18(0,13)$ & $-0,39 * *(0,14)$ \\
\hline Communes de type IV & & & & Réf. & Réf. & Réf. \\
\hline Indicatrices de zones d'emploi & non & non & non & non & oui & oui \\
\hline \multicolumn{7}{|l|}{ Logement } \\
\hline Part des propriétaires & & & & & & $0,66 *(0,35)$ \\
\hline Part de locataires privés & & & & & & Réf. \\
\hline Part de locataires publics & & & & & & $0,75^{*}(0,44)$ \\
\hline \multicolumn{7}{|l|}{ Politique locale d'emploi } \\
\hline Proportion de salariés en emploi aidé & & & & & & $-0,95 *(0,58)$ \\
\hline Nombre d'observations & 1075 & 1075 & 1075 & 1075 & 1075 & 1075 \\
\hline Critère d'information d'Akaike & 3201,97 & 3204,55 & 3189,22 & 3188 & 3290,52 & 3107,81 \\
\hline $\mathrm{R}^{2}$ & 0,34 & 0,34 & 0,35 & 0,36 & 0,40 & 0,44 \\
\hline Log de vraisemblance & $-1597,98$ & $-1597,28$ & $-1589,61$ & $-1583,99$ & $-1523,11$ & $-1515,91$ \\
\hline Test de Breusch-Pagan & 0,47 & & & & & $140,25 * * *$ \\
\hline
\end{tabular}

Sources : FHS de Pôle Emploi 2006, DADS 2002-2005 et recensement 1999 de l'Insee.

Lecture : *** : significatif à $1 \%$; ** : à $5 \%$; : à $10 \%$, écarts-types entre parenthèses.

Estimations réalisées sous GeoDa. Nous utilisons une matrice de type reine à l'ordre 3 pour définir la matrice de poids. 



\section{Hiérarchie des effets}

Les estimations précédentes permettent de mettre en avant les effets propres des deux types de mésappariement, de la ségrégation résidentielle ou encore du dynamisme de l'emploi local, mais elles ne permettent en revanche pas de dire lequel de ces effets prévaut. S'il importe de montrer que ces phénomènes ont effectivement chacun un effet sur la durée de chômage, il est également nécessaire de pouvoir les hiérarchiser, afin de déterminer sur le(s)quel(s) accentuer les efforts au niveau des politiques à mettre en place.

Pour ce faire, nous proposons une méthode qui permet d'examiner l'influence de plusieurs groupes de variables de manière séquentielle. On espère ainsi juger de l'importance relative de l'ajout d'un groupe de variables explicatives sur la qualité de la prédiction du modèle. Concrètement, à l'aide de modèles SEM, nous estimons l'effet de chacun des groupes de variables et nous conservons les valeurs prédites pour la durée de chômage dans la commune. Pour analyser la pertinence d'un groupe de variable, nous regardons le coefficient de corrélation entre les valeurs prédites par le modèle et les valeurs observées. Une valeur élevée du coefficient indique, par exemple, que le groupe de variables retenu explique bien les durées de chômage observées. Dans un premier temps, nous analysons chaque groupe isolément et dans un deuxième temps nous les introduisons successivement, selon des ordres différents.

Le Tableau 5 présente les coefficients de corrélation entre les valeurs prédites par chacun de nos groupes de variables et les valeurs observées, pour différents modèles. Les trois modèles présentés se distinguent par l'ordre d'introduction des groupes. En mettant en oeuvre différentes spécifications, on s'assure que les corrélations obtenues pour un groupe particulier ne sont pas dues à l'ordre dans la hiérarchie des entrées.

La première colonne présente les coefficients de corrélations obtenus pour chacun des groupes de variables introduits isolément dans la régression. Il ressort de ce procédé que les mécanismes les plus pertinents dans l'explication des disparités de durées de chômage sont le mauvais appariement des compétences, les effets de ségrégation et le mauvais appariement spatial, ce qui confirme nos résultats antérieurs. A nouveau, on note que les indicatrices de zones d'emplois sont les variables les plus explicatives du modèle (elles affichent le coefficient de corrélation le plus élevé entre les valeurs prédites et les valeurs observées). Ce résultat tend à montrer qu'une forte hétérogénéité entre les zones d'emplois demeure encore inexpliquée dans notre modèle, et qu'un certain nombre d'effets restent encore non identifiés.

La seconde colonne présente les évolutions successives des coefficients de corrélation suite à l'introduction séquentielle de groupes de variables supplémentaires, tandis que la dernière colonne présente la variation du coefficient attribuée à l'ajout du groupe de variable mentionné. Nous retrouvons que les trois mécanismes qui contribuent le plus à l'augmentation du pouvoir explicatif du modèle sont ceux qui étaient déjà identifiés comme les plus pertinents en termes de portée explicative et, dans une moindre mesure, les variables propres au dynamisme local de l'emploi. Les variables mesurant le logement ou les politiques locales d'emploi semblent jouer un rôle plus marginal. 
Tableau 5. Résultats des corrélations entre valeurs observées et valeurs prédites

\begin{tabular}{lccc}
\hline & $\begin{array}{c}\text { Régressions } \\
\text { multivariées }\end{array}$ & \multicolumn{2}{c}{ Entrées hiérarchiques } \\
\hline Modèle 1 & Corrélations & Corrélations & Corrélations \\
\hline Effets de ségrégation & $0,145^{* * *}$ & $0,145^{* * *}$ & \\
Mauvais appariement spatial & $0,268^{* * *}$ & $0,194^{* * *}$ & 0,049 \\
Mauvais appariement des compétences & $0,140^{* * *}$ & $0,269^{* * *}$ & 0,075 \\
Dynamisme local de l'emploi & $0,083^{* * *}$ & $0,273^{* * *}$ & 0,004 \\
Logement & $0,055^{*}$ & $0,274^{* * *}$ & 0,001 \\
Politique locale d'emploi & 0,013 & $0,277^{* * *}$ & 0,003 \\
Zones d'emplois & $0,419^{* * *}$ & $0,531^{* * *}$ & 0,254 \\
\hline Modèle 2 & Corrélations & Corrélations & Corrélations \\
\hline Mauvais appariement spatial & $0,140^{* * *}$ & $0,140^{* * *}$ & \\
Mauvais appariement des compétences & $0,268^{* * *}$ & $0,285^{* * *}$ & 0,145 \\
Dynamisme local de l'emploi & $0,083^{* *}$ & $0,289^{* * *}$ & 0,004 \\
Effets de ségrégation & $0,145^{* * *}$ & $0,271^{* * *}$ & $-0,018$ \\
Politique locale d'emploi & 0,013 & $0,274^{* * *}$ & 0,003 \\
Logement & $0,055^{*}$ & $0,277^{* * *}$ & 0,003 \\
Zones d'emplois & $0,419^{* * *}$ & $0,531^{* * *}$ & 0,254 \\
\hline Modèle 3 & Corrélations & Corrélations & Corrélations \\
\hline Mauvais appariement des compétences & $0,268^{* * *}$ & $0,268^{* * *}$ & \\
Effets de ségrégation & $0,145^{* * *}$ & $0,194^{* * *}$ & $-0,074$ \\
Mauvais appariement spatial & $0,140^{* * *}$ & $0,269^{* * *}$ & 0,075 \\
Logement & $0,055^{*}$ & $0,270^{* * *}$ & 0,001 \\
Dynamisme local de l'emploi & $0,083^{* *}$ & $0,273^{* * *}$ & 0,003 \\
Politique locale d'emploi & 0,013 & $0,277^{* * *}$ & 0,004 \\
Zones d'emplois & $0,419^{* * *}$ & $0,531^{* * *}$ & 0,254 \\
\hline
\end{tabular}

Sources : FHS de Pôle Emploi 2006, DADS 2002-2005 et recensement 1999 de l'Insee.

Niveaux de significativité : *** au seuil de $1 \%$; ** au seuil de 5\%; * au seuil de $10 \%$.

Notes : le coefficient de 0,145 pour le groupe de variables "Effets de ségrégation" signifie que la corrélation entre les valeurs prédites par le modèle économétrique (modèle de type SEM) avec ces seules variables explicatives et les valeurs observées est de 0,145 .

Estimations réalisées sous GeoDa. Nous conservons une matrice de type reine à l'ordre 3 pour définir la matrice de poids.

Ainsi, ces tests empiriques confirment que les phénomènes de mésappariement spatial et de mésappariement des compétences sont parmi les plus pertinents à considérer dans l'explication des écarts observés des durées de chômage. Dès lors, se pose la question des politiques publiques à mettre en place pour lutter contre ces problèmes d'appariement et favoriser le retour à l'emploi. 


\section{Conclusions}

Si l'on souhaite expliquer l'ampleur des disparités locales dans les durées de chômage, une fois contrôlées les caractéristiques des chômeurs, on peut distinguer deux ensembles de facteurs : à compétences données, la distance physique entre les chômeurs et les emplois peut expliquer la difficulté à retrouver un emploi (mauvais appariement spatial); à distance physique donnée, l'inadéquation des compétences des chômeurs aux besoins des entreprises peut hypothéquer la réinsertion sur le marché du travail (mauvais appariement des compétences).

Ces deux facteurs explicatifs n'impliquent pas des recommandations équivalentes en termes de politique publique. Dans le premier cas, les principaux leviers s'appuient notamment sur une régulation du marché locatif, avec une répartition stratégique du logement social, sur des politiques de transport adaptées ou sur la fourniture de biens publics locaux. Dans le second cas, la stratégie consiste plutôt à développer des politiques de formation ou à réduire le coût du travail par des incitations fiscales à l'embauche de chômeurs dont les compétences ne correspondent pas parfaitement aux postes de travail.

Afin de tester la pertinence de ces différents leviers, nous avons développé une application empirique cherchant à déterminer la pertinence et le poids relatif des différents facteurs explicatifs du chômage local. Cette approche nous conduit à la conclusion suivante : l'origine des fractures spatiales doit être conjointement cherchée du côté de la distance physique aux emplois, de la mauvaise adéquation entre compétences des travailleurs et qualification des postes, et de la ségrégation de certains quartiers qui souffrent d'un déficit collectif de réseaux d'embauche, dans un pays où seules $30 \%$ des offres d'emploi passent par Pôle Emploi. Face à ce cumul des inégalités sur le marché du travail, l'efficacité supposée des différentes politiques mises en œuvre dépend de la cause prédominante du chômage sur chaque bassin local d'emploi.

L'application empirique que nous avons proposée sur des données franciliennes confirme la coexistence de plusieurs types de mésappariement : difficultés d'appariement liées à la distance aux emplois, aux qualifications requises, et au voisinage, qui se renforcent l'une l'autre. Nos résultats tendent à montrer que les politiques les plus efficaces pour réduire la durée du chômage et la fracture spatiale sont celles qui tendent, d'une part, à réduire le mauvais appariement entre les compétences requises par les entreprises et celles proposées par les demandeurs d'emplois et, d'autre part, celles qui réduisent le mauvais appariement spatial entre les emplois et les demandeurs d'emplois. 


\section{BIBLIOGRAPHIE}

Anselin L. (2006). Spatial Regression, Document du National Center for Supercomputing Applications, University of Illinois.

Brueckner J. et Martin R. (1997). "Spatial Mismatch: an Equilibrium Analysis", Regional Science and Urban Economics, n²7, pp. 693-714.

Brunet C., Clark A. et Lesueur J-Y. (2007). "Statut résidentiel et durée de chômage en France et au Royaume-Uni : des faits stylisés aux résultats micro économétriques", Revue Française d'Economie, vol. 22, n², pp. 165-190.

Brunet C. et Lesueur J-Y. (2003). "Le statut résidentiel affecte-t-il la durée de chômage ? Une estimation micro-économétrique sur données françaises", Revue Economique, vol. 55, n³, pp. 569578.

Brunet C. et Lesueur J-Y. (2009). "Do Homeowners Stay Unemployed Longer? A French Microeconometric Study", in Homeownership and the labour market in Europe, Oxford University Press.

Buisson M.A. et Mignot D. (dir.). (2005). Concentration économique et ségrégation spatiale, De Boeck, Collection «Economie, Société, Région ».

Cavaco S. et Lesueur J.Y. (2002). "Contraintes spatiales et durée de chômage”, Revue Française d'Economie, vol. 18, n³, pp. 229-257.

Crane J. (1991). "The Epidemic Theory of Ghettos and Neighborhood Effects on Dropping-Out and Teenage Child-Bearing", American Journal of Sociology, vol. 96, pp. 1126-1259.

Davezies L. (2007). Croissance sans développement en Île-de-France, Rapport pour la Caisse des Dépôts.

Davis S.J. et Haltiwanger J. (1990). "Gross Job Creation and Destruction: Microeconomic Evidence and Macroeconomic Implications”, NBER Macroeconomics Annual.

Dietz R. (2002). "The Estimation of Neighborhood Effects in the Social Sciences: An Interdisciplinary Approach », Social Science Research, vol. 31, pp. 539-575.

Duguet E., Goujard A. et L'Horty Y. (2007). "Les inégalités territoriales d'accès à l'emploi : une analyse cartographique à partir de sources exhaustives", Economie et Statistique, n 415-416, pp. 17-44.

Duguet E., L'Horty Y. et Sari F. (2009). "Sortir du chômage en Île-de-France : disparités territoriales, spatial mismatch et ségrégation résidentielle", Revue Economique, vol. 60, n4, pp. 9791010.

Duhautois R. (2002). "Les réallocations d'emplois en France sont-elles en phase avec le cycle?", Economie et Statistique, $\mathrm{n}^{\circ}$ 351, pp. 87-103.

Dujardin C., Selod H. et Thomas I. (2004). "Le chômage dans l'agglomération bruxelloise : une explication par la structure urbaine", Revue d'Economie Régionale et Urbaine, février, pp. 3-28.

Forslund A., Johansson P. et Lindqvist L. (2004). "Employment subsidies - A fast lane from unemployment to work?", Working paper 2004-18, IFAU, Uppsala.

Gavrel F., Georges N., L'Horty Y. et Lebon I. (2012). "Inadéquation des qualifications et fracture spatiale", mimeo.

Galster G. (2008). "Quantifying the effect of neighbourhood on individuals: challenges, alternative approaches and promising direction”, Schmollers Jahrbuch, vol. 128, n ${ }^{\circ}$, pp. 1-42. 
Gaschet F. et Gaussier N. (2003). "Ségrégation urbaine et marchés du travail au sein de l'aire urbaine bordelaise: quelle portée pour l'hypothèse de mauvais appariement spatial ?", Cahiers du GRES n¹4, décembre.

Gobillon L. et Selod H. (2007). "Ségrégation résidentielle, accessibilité aux emplois, et chômage : le cas de l'Île-de-France", Economie et Prévision, n 180-181, pp. 19-38.

Hellerstein J., Neumark D. et McInemey M. (2008). "Spatial Mismatch or Racial Mismatch ?", Journal of Urban Economics, vol. 64, pp. 464-479.

Holzer H. (1996). What Employers Want: Job Prospects for Less-Educated Workers, New York: Russell Sage Foundation, 182 pages.

Holzer H., Quigley J. et Raphael S. (2003). "Public Transit and the Spatial Distribution of Minority Employment: Evidence from a Natural Experiment", Journal of Policy Analysis and Management, n²2, pp. 415-442.

Ihlanfeldt K. et Sjoquist D. (1998). "The Spatial Mismatch Hypothesis: A Review of Recent Studies and Their Implications for Welfare Reform”, Housing Policy Debate, n9 9, pp. 849-892.

Immergluk D. (1998). "Job Proximity and the Urban Employment Problem: Do Suitable Nearby Jobs Improve Neighbourhood Employment Rates?”, Urban Studies, n³5, pp. 7-23.

Jayet H. (1993). Analyse Spatiale Quatitative, Une Introduction, Paris, Economica, 206 pages.

Kain J. (1968). "Housing Segregation, Negro Employment, and Metropolitan Decentralization", Quarterly Journal of Economics, n`82, pp. 175-197.

Kawabata M. (2003). "Job Access and Employment among Low-Skilled Autoless Workers in US Metropolitan Areas", Environment and Planning A, n³5, pp. 1651-1658.

Layard R., Jackman R. et Savouri S. (1990). "Labour Market Mismatch: a Framework of Though", in Mismatch and Labor Mobility, Paoda-Schioppa F. (eds), Cambridge University Press.

Le Gallo J. (2002). "Économétrie spatiale : l'auto-corrélation spatiale dans les modèles de régression linéaire", Économie et Prévision, n 155, p. 139-157.

LeSage J.P. (1998). "Spatial Econometrics », Université de Tolède, Mimeo.

Moran P. (1948). "The Interpretation of Statistical Maps", Journal of the Royal Statistical Society, vol.10, n², pp. 243-251.

Oswald A. (1996). "A Conjecture on the Explanation for High Unemployment in the Industrialised Nations: Part 1", University of Warwick Economic Research Papers, vol. 475.

Oswald A. (1999). “The Housing Market and Europe's Unemployment: a Non Technical Paper", (unpublished paper).

Pastor J. et Marcelli E. (2000). "Men in the Hood: Skill, Spatial and Social Mismatches among male workers in Los Angeles County", Urban Geography, vol. 21, n6, pp. 474-496.

Patacchini E. et Zenou Y. (2006). " Search Intensity, Cost of Living and Local Labor Markets in Britain”, Regional Science and Urban Economics, vol. 36, pp. 227-248.

Picart C. (2008). "Flux d'emploi et de main-d'œuvre en France : un réexamen", Economie et Statistique, $\mathrm{n}^{\circ} 412$, pp. 27-56.

Raphael S. et Stoll M. (2001). "Can Boosting Minority Car-Ownership Rates Narrow Inter-Racial Employment Gaps?", in Brooking-Wharton Papers on Urban Economic Affairs $n^{\circ} 2$, William G. Gale and Janet Rothenberg Pack (eds), The Brookings Institution: Washington, DC, pp. 99-145.

O'Regan K. (1993). "The Effect of Social Networks and Concentrated Poverty on Black and Hispanic Youth Unemployment”, Annals of Regional Science, vol. 27, pp. 327-342. 
Rogers C. (1997). "Job Search and Unemployment Duration: Implications for the Spatial Mismatch Hypothesis", Journal of Urban Economics, n42, pp. 109-132.

Selod H., Zenou Y. (2006). "City Structure, Job Search and Labour Discrimination: Theory and Policy Implications”, Economic Journal, vol. 116, pp. 1057-1087.

Sneessens H. et Shadman-Mehta F. (1995). "Real Wages, Skill Mismatch and Unemployment Persistence France, 1962-1989”, Annales d'Économie et de Statistique, n 37/38, pp. 255-292. 
12-1. Etre meilleur Apprenti de France : Quels effets sur l'accès à l'emploi ? Les enseignements de deux expériences contrôlées sur des jeunes d'Ile-de-France Pascale Petit, Florent Fremigacci, Loïc du Parquet, Guillaume Pierne

12-2. L'intermédiation financière dans l'analyse macroéconomique : Le défi de la crise Eleni Iliopoulos, Thepthida Sopraseuth

12-3. Evaluer un dispositif sectoriel d'aide à l'emploi : L'exemple des hôtels cafés restaurants de 2004 à 2009

Mathieu Bunel

12-4. Evaluer les réformes des exonérations générales de cotisations sociales Mathieu Bunel, Céline Emond, Yannick L'Horty

12-5. Discrimination à l'embauche des jeunes en Ile-de-France : un diplôme plus élevé compense-t-il une origine maghrébine ?

Emilia Ene

12-6. L'accès à l'emploi après un CAP ou un baccalauréat professionnel : Une évaluation expérimentale

Florent Fremigacci, Yannick L'Horty, Loïc du Parquet, Pascale Petit 
11-1. Les effets du lieu de résidence sur l'accès à l'emploi: Une expérience contrôlée sur des jeunes qualifiés en Ile-de-France

Yannick L'Horty, Emmanuel Duguet, Loïc du Parquet, Pascale Petit, Florent Sari

11-2. Comment développer les emplois favorables à la biodiversité en Ile-De-France ? Jean de Beir, Céline Emond, Yannick L'Horty, Laëtitia Tuffery

11-3. Être mobile pour trouver un emploi ?Les enseignements d'une expérimentation en région parisienne

Loïc du Parquet, Emmanuel Duguet, Yannick L’Horty, Pascale Petit, Florent Sari

11-4. Ce que font les villes pour les ménages pauvres. Résultats d'une enquête nationale sur les communes de plus de 20000 habitants

Denis Anne, Céline Emond, Yannick L’Horty

11-5. Discriminations à l'embauche des jeunes franciliens et intersectionalité du sexe et de l'origine: Les résultats d'un testing

Pascale PETIT, Emmanuel DUGUET, Yannick L'HORTY, Loïc du PARQUET, Florent

SARI

11-6. Les effets du bénévolat sur l'accès à l'emploi. Une expérience contrôlée sur des jeunes qualifiés d'Ile-de-France

Jonathan Bougard, Thomas Brodaty, Céline Emond, Yannick L’Horty, Loïc du Parquet et

Pascale Petit

11-7. « 10000 permis pour réussir ». Evaluation quantitative

Yannick L’Horty, Emmanuel Duguet, Sophie Kaltenmark, Pascale Petit

11-8. Why is there a faster return to work near the border?

Jonathan Bougard

11-9. Evaluer l'impact d'un micro-programme social : une étude de cas expérimentale Yannick L'Horty, Emmanuel Duguet, Pascale Petit

11-10. Les effets des aides publiques aux Hôtels Cafés Restaurants et leurs interactions : Une évaluation sur micro-données d'entreprises

Mathieu Bunel, Yannick L'Horty

11-11. Pourquoi tant de chômeurs à Paris

Yannick L'Horty, Florent Sari

11-12. LE WIKI IO : Réduire les risques de décrochage et d'abandon à la sortie du collège

Solène Coursaget, Emmanuel Duguet, Yannick L'Horty, Pascale Petit, Emmanuel Quenson

11-13. Le grand Paris de l'emploi

Yannick L'Horty, Florent Sari

11-14. Quelle politique publique pour protéger la biodiversité Jean De Beir, Céline Emond, Yannick L'Horty, Laetitia Tuffery 
La Fédération de recherche CNRS Travail, Emploi et Politiques Publiques (TEPP, FR $\mathbf{n}^{\circ} \mathbf{3 1 2 6}$ ) réunit des centres de recherche en économie et sociologie :

- Le Centre d'Etudes des Politiques Economiques de l'université d'Evry, EPEE, Université d'Evry Val d'Essonne

- Le Centre Pierre Naville, CPN, Université d'Evry Val d'Essonne

- Le Centre de Recherche en Economie et Management, CREM, Université de Caen Basse Normandie et Université de Rennes 1

- L'Equipe de Recherche sur les Marchés, l'Emploi et la Simulation, ERMES, Université deParis II Panthéon-Assas

- L'Equipe de Recherche sur l'Utilisation des Données Temporelles en Economie, ERUDITE, Université de Paris-Est Créteil et Université de Paris-Est Marne-la-Vallée

- Le Groupe d'Analyse des Itinéraires et des Niveaux Salariaux, GAINS, Université du Maine

La Fédération TEPP rassemble 150 chercheurs et enseignants-chercheurs, 140 doctorants et 40 chercheurs associés, qui étudient les mutations du travail et de l'emploi en relation avec les choix des entreprises et analysent les politiques publiques en mobilisant les nouvelles méthodes d'évaluation. 